\title{
Reduction of State-to-State to Macroscopic Models for Hypersonics
}

\author{
A. Bourdon ${ }^{*}, 1,2$, J. Annaloro ${ }^{3}$, A. Bultel ${ }^{3}$, M. Capitelli ${ }^{4}$, G. Colonna ${ }^{5}$, A. Guy ${ }^{1,2}$, T.E. Magin ${ }^{6}$, \\ A. Munafó ${ }^{6}$, M.Y. Perrin ${ }^{1,2}$ and L.D. Pietanza ${ }^{5}$
}

${ }^{I}$ CNRS, UPR 288 “Laboratoire d'Energétique Moléculaire et Macroscopique, Combustion" (EM2C), Grande voie des vignes, 92295 Châtenay-Malabry, France

${ }^{2}$ Ecole Centrale Paris, Grande voie des vignes, 92295 Châtenay-Malabry, France

${ }^{3}$ CORIA, UMR CNRS 6614, Université de Rouen, BP 12, Avenue de l'Université, 76801 Saint-Etienne du Rouvray Cedex, France

${ }^{4}$ Dip. di Chimica, Universitá di Bari, Via Orabona, 4, 70126, Italy

${ }^{5}$ CNR-IMIP, sede di Bari, Via Amendola 122/D, 70126, Italy

${ }^{6}$ Aeronautics and Aerospace Department, von Karman Institute for Fluid Dynamics, Chaussée de Waterloo 72, 1640 Rhode-Saint-Genèse, Belgium

\begin{abstract}
Four different types of macroscopic models developed for the vibration-chemistry coupling in nonequilibrium flows for re-entry applications are presented. First, using an approach based on nonequilibrium thermodynamics, global rate coefficients of dissociation of $\mathrm{N}_{2}$ and $\mathrm{O}_{2}$ under parent molecular or atomic impact and backward molecular recombination are determined. Then a Two-Level Distribution (TLD) model is developed, in which a relaxation equation for vibrational temperature is solved as in the case of multi-temperature models but with the simultaneous solution of a kinetic equation, as in the case of state-to-state models, but only for the last vibrational level. In a third approach, a multiinternal temperature model is presented to describe accurately the vibrational distribution function in using several groups of levels, within which the levels are assumed to follow a Boltzmann distribution at an internal temperature of the group. This multi-internal temperature model allows us to describe accurately the vibrational energy relaxation and dissociation processes behind a strong shock wave. Finally, a rovibrational collisional coarse-grain model is developed to reduce a detailed rovibrational mechanism for the internal energy excitation and dissociation processes behind a strong shock wave in a nitrogen flow.
\end{abstract}

Keywords: Macroscopic models, nonequilibrium flows, re-entry applications, state-to-state models, vibration-chemistry coupling.

\section{INTRODUCTION}

The planetary atmospheric entry phase of a moving body (spacecraft, probe, meteorite, etc.) involves a complex chemistry mainly due the weak values of the hydrodynamic characteristic time scale with respect to the characteristic time scales required to reach chemical equilibrium. The most accurate way to simulate nonequilibrium flows for re-entry applications is to use State-to-State models (STS), in which a system of master equations is solved, with one equation for each internal energy level of each species. This approach allows a self consistent calculation of chemistry and energy source terms without any assumption on the internal distribution. In the last years, STS vibrational kinetics have been developed and applied to 1D high enthalpy flows, such as boundary layer [1,2], nozzle expansion [3-6] and shock

*Address correspondence to this author at the CNRS, UPR 288 "Laboratoire d'Energétique, Moléculaire et Macroscopique, Combustion" (EM2C), Grande voie des vignes, 92295 Châtenay-Malabry, France;

Tel: 33 (0)1 411310 46; Fax: 33 (0)1 470280 35;

E-mail: anne.bourdon@ecp.fr wave $[3-5,7-11]$. These models give a very accurate insight of the behavior of chemical processes and predict, for example, in recombination regimes, the overpopulation of the tail of vibrational distributions due to preferential pumping of high-lying vibrational levels [12]. Unfortunately, the systematic implementation of STS is difficult due to both the lack of data for rate coefficients for each internal level and to long computational times, preventing the use of these models in higher (2D-3D) dimensional codes. For these reasons, one of the most commonly used approach, especially in the case of $2 \mathrm{D}$ or $3 \mathrm{D}$ high enthalpy flow simulations for re-entry applications, is the MultiTemperature (MT) approach [13-19]. In MT, each degree of freedom is described by a Boltzmann distribution at a different temperature. As an example, for diatomic molecules, the rotational degree of freedom is generally assumed to be in equilibrium with the translational temperature while the vibrational temperature is determined solving a linear relaxation equation. Vibrational temperatures affect the rate coefficients for chemical reactions, such as molecular dissociation, which can be accelerated by vibrational excited distributions. One of the most widely 
used multitemperature model has been proposed by Park [13-15]. In this model, the rate coefficients are calculated using an Arrhenius function depending on an effective temperature calculated as the geometric average of the translational and vibrational temperatures. However, as discussed by Park [16, 20], even if very efficient from a computational point of view, the use of multitemperature models can only be justified when the departure from the Boltzmann population is small (i.e., for low velocity and high pressure reentry conditions). Moreover, multitemperature rate coefficients have been obtained heuristically, to reproduce experimental data obtained in shock tube facilities.

To overcome this problem, the group of St. Petersburg proposed to approximate the vibrational distribution by a piecewise function dividing the vibrational ladder in three regions [21]. The low energy region is approximated by a Treanor distribution, the middle energy range by a constant function and the distribution tail by a Boltzmann function. This approximation is based on the idea that the low and middle levels are determined mainly by mono-quantum Vibration-Vibration (VV) processes and high energy levels by mono-quantum Vibration-Translation (VT) processes. These assumptions are based on the general hierarchy of relaxation times for the different processes. It is interesting to note that recently, significant efforts have been done by different research groups at NASA Ames [22, 23], University of Barcelona [24] and Plasma Institute in Bari [25-27] to calculate rovibrational rate coefficients for processes of importance for re-entry applications. In these new databases, the importance of multi-quantum VT and dissociation/recombination processes has been clearly put forward. Then there is the need to derive new macroscopic models to carry out $1 \mathrm{D}, 2 \mathrm{D}$ and $3 \mathrm{D}$ simulations of high enthalpy flows for re-entry applications which allow to better describe nonequilibrium flows and which are based on most recent state-to-state rate coefficients. In this paper, we discuss the derivation of different types of macroscopic models and we focus on the modeling of the vibrationchemistry coupling. In Section 2, we first present global dissociation and recombination rate coefficients, very easy to implement in multi-dimensional flow codes. In Section 3, we present a Two-Level Distribution (TLD) model, in which a relaxation equation for vibrational temperature is solved as in the case of MT but with the simultaneous solution of a kinetic equation, as in the case of STS, but only for the last vibrational level. In Section 4, we propose to better represent the vibrational nonequilibrium of a molecular flow, in using several internal vibrational temperatures. Finally in Section 5 , we discuss the derivation of a coarse-grain model from a detailed rovibrational database.

\section{GLOBAL DISSOCIATION AND RECOMBINATION RATE COEFFICIENTS}

In this section, we propose to study global rate coefficients. We illustrate their determination for $\mathrm{N}_{2}$ and $\mathrm{O}_{2}$ dissociation under heavy particle impact. The developed method is also applied to recombination. The relation between these rate coefficients and the equilibrium constant is discussed.

\subsection{Global Rate Coefficient Definition}

The balance equation for a species $X_{n}(n=1$ for atoms and $n=2$ for diatomic molecules) in its excited state $i$ in a volume $V$ without inner swirls and without radiative phenomena can be written under the form

$\frac{1}{V} \frac{d N_{X_{n, i}}}{d t}=\left[\dot{X}_{n, i}\right]_{C}$

where $N_{X_{n, i}}=\left[X_{n, i}\right] V$ is the number of particles $X_{n, i}$ and $\left[X_{n, i}\right]$ their number density. Inside $V$, elementary processes occur which can be generally written as

$X_{n, i}+P \rightleftarrows X_{n-\alpha, j}^{\beta+}+\alpha X_{1, m}+\beta e^{-}+P$

where $P$ is the collision partner and $(n, \alpha, \beta)$ defines the type of the elementary process. The process is called elementary since it refers to excited states only. If initially the total number of $X_{n}$ is higher than its value at equilibrium $\left(N_{X_{n}}>\left(N_{X_{n}}\right)_{e q}\right)$, the system globally evolves according to the process

$X_{n}+P \stackrel{f}{\rightarrow} X_{n-\alpha}^{\beta+}+\alpha X_{1}+\beta e^{-}+P$

This global process is denoted $f$. The temporal evolution of $N_{X_{n}}$ is therefore governed by

$\frac{1}{V}\left(\frac{d N_{X_{n}}}{d t}\right)_{f}=\frac{1}{V} \sum_{i} \frac{d N_{X_{n, i}}}{d t}=\left[\dot{X}_{n}\right]_{C}$

which is similar to Eq. (1), but for the species $X_{n}$ for all excited states. The global rate coefficient $k_{f}$ is then defined as the parameter depending on $T_{P}$ the kinetic temperature of $\mathrm{P}$, such as

$\frac{1}{V}\left(\frac{d N_{X_{n}}}{d t}\right)_{f}=-k_{f}\left[X_{n}\right][P]$.

The global rate coefficient is therefore the equivalent or effective rate coefficient reflecting the behaviour of the species $X_{n}[12,28-33]$. This rate coefficient is a measurement of the net variation rate of $X_{n}$ due to the complex behaviour of the excited states.

If $N_{X_{n}}<\left(N_{X_{n}}\right)_{e q}$, elementary processes lead to the global process of type $r$

$X_{n-\alpha}^{\beta+}+\alpha X_{1}+\beta e^{-}+P \stackrel{r}{\longrightarrow} X_{n}+P$

with

$\frac{1}{V}\left(\frac{d N_{X_{n}}}{d t}\right)_{r}=\frac{1}{V} \sum_{i} \frac{d N_{X_{n, i}}}{d t}=k_{r}\left[X_{n-\alpha}^{\beta+}\right]\left[X_{1}\right]^{\alpha}\left[e^{-}\right]^{\beta}[P]$

Owing to the value of $T_{P}$ and the initial conditions, the values of

$k_{f}^{*}=-\frac{1}{\left[X_{n}\right][P]} \frac{1}{V} \sum_{i} \frac{d N_{X_{n, i}}}{d t}$ 
for a process of type $f$ or

$k_{r}^{*}=\frac{1}{\left[X_{n-\alpha}^{\beta+}\right]\left[X_{1}\right]^{\alpha}\left[e^{-}\right]^{\beta}[P]} \frac{1}{V} \sum_{i} \frac{d N_{X_{n, i}}}{d t}$

for a process of type $r$ are time-dependent. The simplest proof is given by the fact that $k_{f, r}^{*} \neq 0$ during the evolution and $k_{f, r}^{*}=0$ when equilibrium is reached since $\frac{d N_{X_{n, i}}}{d t}=0$ $\forall i$. When we follow $k_{f}^{*}$ or $k_{r}^{*}$ in time, a quasi-steady state (QSS) is observed. This behaviour is illustrated in section 2.3. However, experimental determinations of the global rate coefficients show that they are time-independent provided the temperature is steady. The QSS is therefore the only duration for which the concept of global rate coefficient is relevant. As a result, $k_{f}$ and $k_{r}$ are then identified as the values of $k_{f}^{*}$ and $k_{r}^{*}$ during the QSS, respectively.

\subsection{Global Rate Coefficients and Equilibrium Constant}

It is worth noticing that $\sum_{i} \frac{1}{V} \frac{d N_{X_{n, i}}}{d t}$ refers to irreversible processes in (8) and (9). In addition, they depend on initial conditions. In the general case, $k_{f}$ and $k_{r}$ are therefore independent from each other. However, they are often assumed linked by the corresponding equilibrium constant. This peculiarity can be observed only for weak departure from equilibrium. Indeed, during a global process, when $\left(p, T_{P}\right)$ are maintained constant, the time variation of the free enthalpy of the mixture is governed by

$$
\begin{aligned}
& \left(\frac{d G}{d t}\right)_{f, r}=\mu_{X_{n-\alpha}^{\beta+}}\left(p, T_{P}\right) \frac{d N_{X_{n-\alpha}^{\beta+}}^{\beta+}}{d t} \\
& +\alpha \mu_{X_{1}}\left(p, T_{P}\right) \frac{d N_{X_{1}}}{d t}+\beta \mu_{e^{-}}\left(p, T_{P}\right) \frac{d N_{e^{-}}}{d t} \\
& +\mu_{X_{n}}\left(p, T_{P}\right) \frac{d N_{X_{n}}}{d t}+\frac{d N_{X_{n-\alpha, j}^{\beta+}}}{d t} k_{B} T_{P} \ln x_{X_{n-\alpha, j}^{\beta+}} \\
& +\alpha \frac{d N_{X_{1}}}{d t} k_{B} T_{P} \ln x_{X_{1}}+\beta \frac{d N_{e^{-}}}{d t} k_{B} T_{P} \ln x_{e^{-}} \\
& +\frac{d N_{X_{n}}}{d t} k_{B} T_{P} \ln x_{X_{n}}
\end{aligned}
$$

where $\mu_{l}$ is the chemical potential (free enthalpy) per particle of type $l, x_{l}$ the mole fraction of the species $l$, and $\mu_{l}\left(p, T_{P}\right)=\mu_{l}\left(p, T_{P}, x_{l}\right)-k_{B} T_{P} \ln x_{l}$ the pure species chemical potential assuming a dilute gas-like behaviour (see [34]) with $k_{B}$ the Boltzmann constant. Defining the extent of reaction $\xi_{f, r}, K_{f, r}, \delta=1$ for a reaction of type $f, \delta=-1$ for a reaction of type $r$, and $\Delta \mu\left(p, T_{P}\right)$ such as

$$
\begin{gathered}
\left(\frac{d \xi}{d t}\right)_{f, r}=\delta\left(\frac{d N_{X_{n-\alpha}^{\beta+}}}{d t}\right)_{f, r}=\delta\left(\frac{d N_{X_{1}}}{d t}\right)_{f, r} \\
=\delta\left(\frac{d N_{e^{-}}}{d t}\right)_{f, r}=-\delta\left(\frac{d N_{X_{n}}}{d t}\right)_{f, r}, \\
K_{f, r}=\frac{\left[X_{n-\alpha}^{\beta+}\right]\left[X_{1}\right]^{\alpha}\left[e^{-}\right]^{\beta}}{\left[X_{n}\right]}, \\
\Delta \mu\left(p, T_{P}\right)=\mu_{X_{n-\alpha}^{\beta+}}\left(p, T_{P}\right)+\alpha \mu_{X_{1}}\left(p, T_{P}\right)+ \\
\beta \mu_{e^{-}}\left(p, T_{P}\right)-\mu_{X_{n}}\left(p, T_{P}\right),
\end{gathered}
$$

$K_{f, r}$ finally writes as

$K_{f, r}=e^{\frac{1}{k_{B} T_{P}}\left[\delta\left(\frac{\frac{d G}{d t}}{\frac{d \xi}{d t}}\right)_{f, r}-\Delta \mu\left(p, T_{P}\right)\right]}$.

When equilibrium is reached, $G$ does not vary anymore, even if a fluctuation of the extent $\xi$ is observed. As a result,

$K_{e q}=e^{\frac{1}{k_{B} T_{P}}\left[-\Delta \mu\left(p, T_{P}\right)\right]}$ and

$K_{f, r}=K_{e q} e^{\frac{\delta}{k_{B} T_{P}}\left(\frac{\frac{d G}{d t}}{\frac{d \xi}{d t}}\right)_{f, r}}$.

The global reaction is irreversible which leads to

$\left(\frac{d G}{d t}\right)_{f, r}<0$ and $\left(\frac{d \xi}{d t}\right)_{f, r}>0$ (see [34]).

Therefore $K_{f}<K_{e q}$ and $K_{r}>K_{e q}$. Let us assume the system close to equilibrium such as $N_{X_{n}}>\left(N_{X_{n}}\right)_{e q}$ in a first part and $N_{X_{n}}<\left(N_{X_{n}}\right)_{e q}$, in another part, the total number being the same as at equilibrium for the complete system. Owing to the weak departure from equilibrium, $N_{X_{n}}$ evolves in each part such as

$\left(\frac{d N_{X_{n}}}{d t}\right)_{f}+\left(\frac{d N_{X_{n}}}{d t}\right)_{r}=0$

From the product $K_{f} K_{r}$, the ratio $\frac{k_{f}}{k_{r}}$ can be deduced

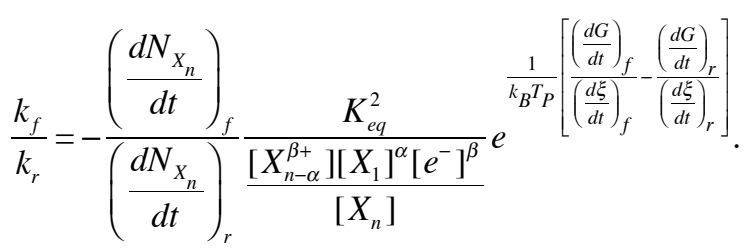

Equilibrium being stable with respect to fluctuations, 


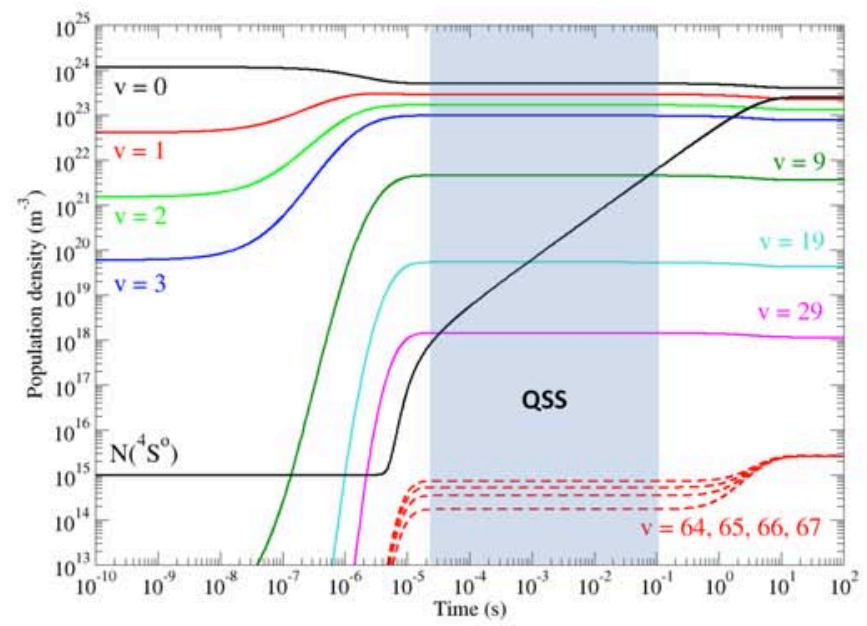

(a)

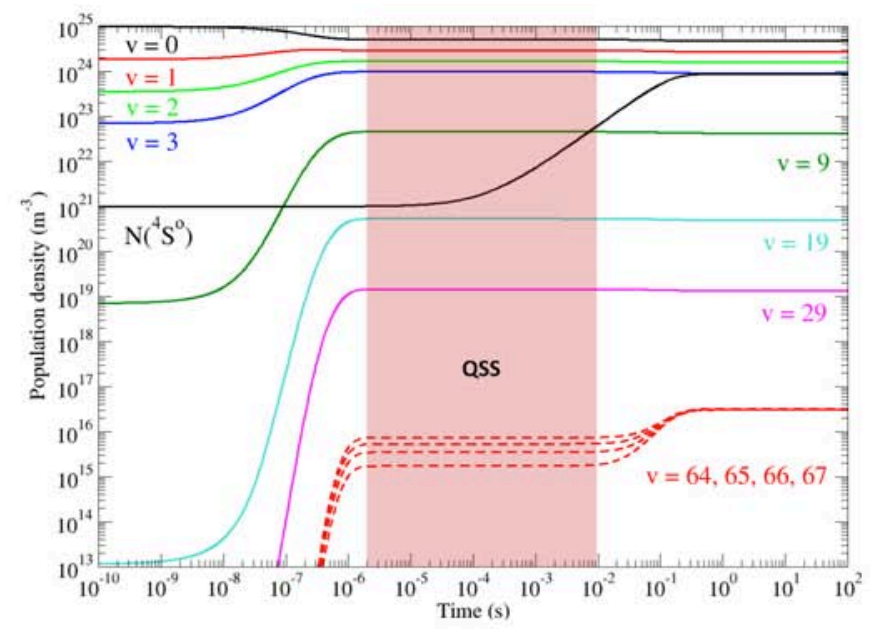

(b)

Fig. (1). Time evolution of the population density of excited states of $\mathrm{N}$ and $\mathrm{N}_{2}$ under $P \equiv \mathrm{N}_{2}$ impact at $T_{A}=6,000 \mathrm{~K}$ in dissociation situations for arbitrary initial conditions with (a) $p=10^{5} \mathrm{~Pa}$ and (b) $p=10^{6} \mathrm{~Pa}$.

$\frac{d G}{d t}=\left(\frac{d G}{d t}\right)_{f}+\left(\frac{d G}{d t}\right)_{r} \equiv 0$

is obvious and using (11) and (16), we obtain, since the system is close to equilibrium

$$
\frac{k_{f}}{k_{r}}=\frac{K_{e q}^{2}}{\frac{\left[X_{n-\alpha}^{\beta+}\right]\left[X_{1}\right]^{\alpha}\left[e^{-}\right]^{\beta}}{\left[X_{n}\right]}} \simeq \frac{K_{e q}^{2}}{\left(\frac{\left[X_{n-\alpha}^{\beta+}\right]\left[X_{1}\right]^{\alpha}\left[e^{-}\right]^{\beta}}{\left[X_{n}\right]}\right)_{e q}}=K_{e q} .
$$

It is worth noticing that $k_{f} / k_{r} \simeq K_{e q}$ is only valid close to equilibrium. Indeed, Eqs. (16) and (18) are not fulfilled in case of strong nonequilibrium.

\subsection{Global Rate Coefficients for $\mathrm{N}_{2}$ and $\mathrm{O}_{2}$ Dissociation and Recombination}

These cases correspond to $X \equiv \mathrm{N}$ or $\mathrm{O}, n=2, \alpha=1$ and $\beta=0$. The vibrational energy diagram of the molecules is taken from [26] and [35]. The cases $P \equiv \mathrm{N}_{2}$ or $\mathrm{N}$ for the dissociation of $\mathrm{N}_{2}$ and $P \equiv \mathrm{O}_{2}$ or $\mathrm{O}$ for the dissociation of $\mathrm{O}_{2}$ are treated. Elementary processes (2) correspond to vibration-translation (when $P \equiv \mathrm{N}_{2}, \mathrm{O}_{2}, \mathrm{~N}$ or $\mathrm{O}$ ) and vibration-vibration (when $P \equiv \mathrm{N}_{2}$ or $\mathrm{O}_{2}$ ) processes. Multiquanta jumps are taken into account only when $P \equiv \mathrm{N}$ or $\mathrm{O}$; for molecule-induced collisions, the rate coefficients for $\Delta v= \pm 1$ transitions are indeed largely higher $[2,26,27,36]$.

Fig. (1a, b) illustrate the time evolution of the species densities in the case of the dissociation of $\mathrm{N}_{2}\left(K_{f}<K_{e q}\right)$ under $P \equiv \mathrm{N}_{2}$ impact when $T_{P} \equiv T_{A}=6,000 \quad K$ for $p=10^{5} \mathrm{~Pa}$ and $p=10^{6} \mathrm{~Pa}$, respectively. Before the final equilibrium state, the mixture reaches an intermediate quasisteady state characterized by constant population densities for excited states. One can see that this QSS is not the same. It therefore depends on the initial conditions and particularly on the pressure level, a magnification of one order of magnitude of the pressure leading to characteristic time scales ten times shorter. We can observe that states close to the dissociation limit are underpopulated with respect to equilibrium with higher values for the case at higher pressure. Fig. (2) displays the parameter $k_{d}^{*}$ similar to $k_{f}^{*}$ of Eq. (8) for the present case of dissociation. We observe the same QSS as the one previously observed on excited states. Although the cases are different, the value obtained for $k_{d}^{*}$ is the same whatever the initial conditions and the pressure level. Since the common parameter between Fig (1a) and Fig. (1b) is only $T_{A}(6,000 \mathrm{~K}$ in the present case), we deduce that the final value of the global rate coefficient is $k_{d} \equiv k_{d}^{*}(Q S S)$ which depends on $T_{A}$ only. In order to further characterize the global process, we calculated two vibrational temperatures defined as the excitation temperature

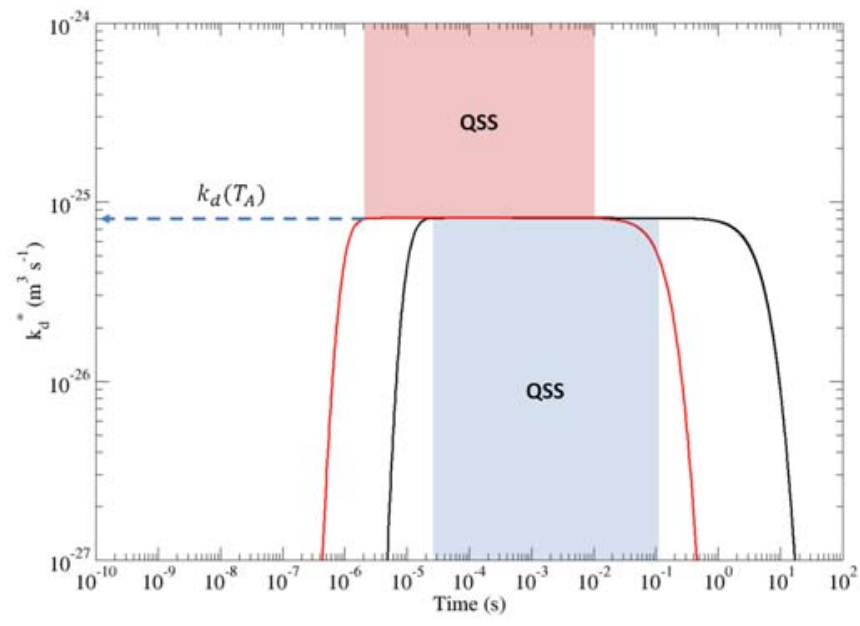

Fig. (2). Evolution of $k_{d}^{*}$ defined as $k_{f}^{*}$ by Eq. (8) in the conditions of Fig. (1a) in black and of Fig. (1b) in red. The QSS corresponding to Fig. $(\mathbf{1} \mathbf{a}, \mathbf{b})$ are indicated. 


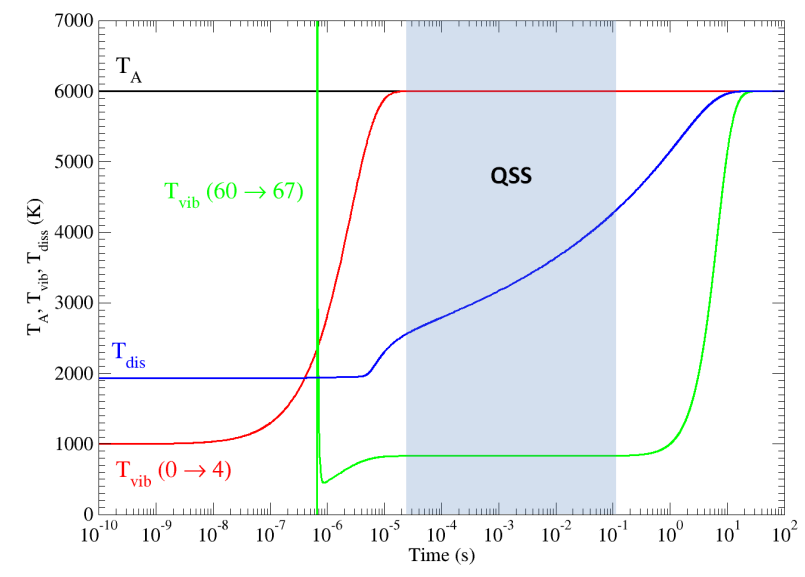

Fig. (3). Evolution of $T_{d i s}, T_{v i b}(0 \rightarrow 4)$ and $T_{v i b}(60 \rightarrow 67)$ in the conditions of Fig. (1a).

$$
T_{v i b}(i \rightarrow j)=-\frac{1}{k_{B}\left[\frac{d}{d E_{v}}\left(\ln \left[N_{2}^{v}\right]\right)\right]_{l s l}}
$$

of the groups corresponding to the first $5(i=0, j=4)$ and to the last $8(i=60, j=67)$ vibrational levels. $l s l$ means that the derivative is the slope of the least square line. On Fig. (3), the different $T_{v i b}$ values (equal to $1,000 \mathrm{~K}$ at $t=0$ ) are compared with $T_{A}$ and the dissociation temperature defined as

$$
T_{d i s}=\frac{E_{d i s}}{k_{B} \ln \left\{\frac{\left[N_{2}^{v=0}\right]}{[N]^{2}} \frac{g_{N}^{2}}{Z_{r}(v=0)}\left(\frac{2 \pi \mu k_{B} T_{A}}{h^{2}}\right)^{3 / 2}\right\}}
$$

with usual notations. $T_{d i s}$ is close to the temperature of the mixture at equilibrium having the same dissociation degree. We can see that the coupling between translation and low lying levels is very rapid contrary to the case of high-lying levels. The evolution of the high-lying levels is so rapid that $T_{v i b}(60 \rightarrow 67)$ is quite different from its initial value for $t>10^{-10} \mathrm{~s}$. This is due to the chosen initial conditions which correspond to a very strong nonequilibrium. Vibrational temperatures are steady during the QSS and $T_{d i s}$ increases since the mixture globally dissociates. The discrepancy between the two vibrational temperatures previously defined illustrates the vibrational nonequilibrium during dissociation, therefore the inequality $K_{f}<K_{e q}$. Dissociation occurs through the last vibrational level since multi-quanta jumps are not taken into account. This level is approximately ten times underpopulated with respect to equilibrium during dissociation. Changing the $T_{v i b}$ initial values leads to a modification of the dynamics of the excited states, but the value of $k_{d}^{*}$ remains unchanged during the QSS and basically depends on $T_{A}$ only. In the present case, the pressure $p$ has no influence on $k_{d}^{*}$ since the plasma is ideal. The previous procedure is repeated for 2,000 $K \leq T_{A} \leq$ $25,000 K$ for $P \equiv \mathrm{N}_{2}$ and $\mathrm{N}$. The $\mathrm{O}_{2}$ dissociation is also treated with $P \equiv \mathrm{O}_{2}$ or $\mathrm{O}$. The results are displayed on Fig. (4). The quasi-linear form of the curves of Fig. (4) suggests an Arrhenius interpolation

$k_{f, r}\left(T_{A}\right)=A_{f, r} T_{A}^{\alpha_{f, r}} e^{-\frac{T_{f, r}}{T_{A}}}$,

the parameters $A_{f}, \alpha_{f}$ and $T_{f}$ of which are listed in Table 1. The resulting rate coefficients can be compared to reference data. Fig. (5) illustrates this comparison for the $\mathrm{N}_{2}$ dissociation under $\mathrm{N}$ impact with the values of [27, 37-41]. The agreement is fairly good.

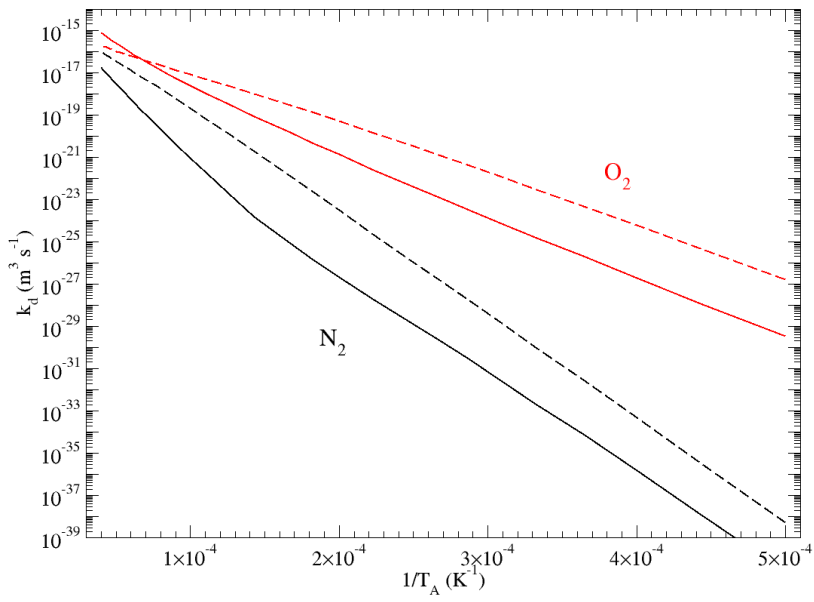

Fig. (4). Global rate coefficient of $\mathrm{N}_{2}$ (in black) and $\mathrm{O}_{2}$ (in red) dissociation for molecular collision partner (continuous lines for $P \equiv \mathrm{N}_{2}$ or $P \equiv \mathrm{O}_{2}$, respectively) and for atomic collision partner (dashed lines for $P \equiv \mathrm{N}$ or $P \equiv \mathrm{O}$, respectively) as a function of $1 / T_{A}$.

The previous approach is applied to the recombination (here performed for $2,000 K \leq T_{A} \leq 6,000 \mathrm{~K}$ only because a clear QSS identification is not systematic for higher temperature). The evolution of $k_{r}$ with $T_{A}$ is satisfactorily interpolated by the Arrhenius form (22). The coefficients $A_{r}$, $\alpha_{r}$ and $T_{r}$ are listed in Table 1. Significant discrepancies are found with literature for the recombination due to molecular impact. For atomic impact, the agreement between our $3 \mathrm{O} \rightarrow \mathrm{O}_{2}+\mathrm{O}$ recombination rate coefficient and the ones due to $[37,41,42]$ is very good. Since $k_{f} \equiv k_{d}$ and $k_{r}$ are independently determined, $k_{d} / k_{r}$ is calculated and compared with the equilibrium (Guldberg \& Waage) constant (see Fig. 6). The discrepancy between $k_{d} / k_{r}$ and $K_{e q}$ remains weak in the case of atomic impact. We have shown in section 2.2 that the ratio is close to $K_{e q}$ when the mixture is close to equilibrium. For $T_{A}=6,000 k$, Figs. $(\mathbf{1 a}, \mathbf{b}, \mathbf{3})$ show that the vibrational distribution is far from equilibrium when $P \equiv \mathrm{N}_{2}$. The ratio $k_{d} / k_{r}$ is therefore far from $K_{e q}$ at $6,000 K$ on Fig. (6). It is interesting to note that in Kustova et al. [21, 43], similar results on global dissociation rates and relations with equilibrium constants have been obtained based on the kinetic theory approach. 
Table 1. $A_{f, r}, \alpha_{f, r}, T_{f, r}$, values for Arrhenius interpolations (22) of the global rate coefficients.

\begin{tabular}{|c|c|c|c|c|c|c|}
\hline \multirow{2}{*}{$\begin{array}{c}\text { Arrhenius Interpolation } \\
\text { Global process }\end{array}$} & \multicolumn{3}{|c|}{$k_{f}=A_{f} T_{A}^{\alpha_{f}} e^{-T_{f} / T_{A}}\left(m^{3} s^{-1}\right)$} & \multicolumn{3}{|c|}{$k_{r}=A_{r} T_{A}^{\alpha_{r}} e^{-T_{r} / T_{A}}\left(m^{6} s^{-1}\right)$} \\
\hline & $A_{f}$ & $\alpha_{f}$ & $T_{f}(K)$ & $A_{r}$ & $\alpha_{r}$ & $T_{r}(K)$ \\
\hline$N_{2}+N_{2} \underset{k_{r}}{\stackrel{k_{f}}{\rightleftarrows}} 2 N+N_{2}$ & $7.61 \times 10^{-34}$ & 4.085 & 96,068 & $2.41 \times 10^{-41}$ & -2.111 & 1430 \\
\hline$N_{2}+N \underset{k_{r}}{\stackrel{k_{f}}{\rightleftarrows}} 3 N$ & $2.62 \times 10^{-11}$ & -0.780 & 115,657 & $1.32 \times 10^{-45}$ & 0.048 & 143 \\
\hline $\boldsymbol{O}_{2}+\boldsymbol{O}_{2} \underset{\boldsymbol{k}_{r}}{\stackrel{\boldsymbol{k}_{f}}{\rightleftarrows}} 2 \boldsymbol{O}+\boldsymbol{O}_{2}$ & $1.23 \times 10^{-25}$ & 2.458 & 58,450 & $1.30 \times 10^{-39}$ & -2.140 & 7448 \\
\hline $\boldsymbol{O}_{2}+\underset{k_{r}}{\stackrel{k_{f}}{\rightleftarrows}} 3 O$ & $2.88 \times 10^{-13}$ & -0.474 & 58,688 & $5.19 \times 10^{-41}$ & -1.141 & -2226 \\
\hline
\end{tabular}

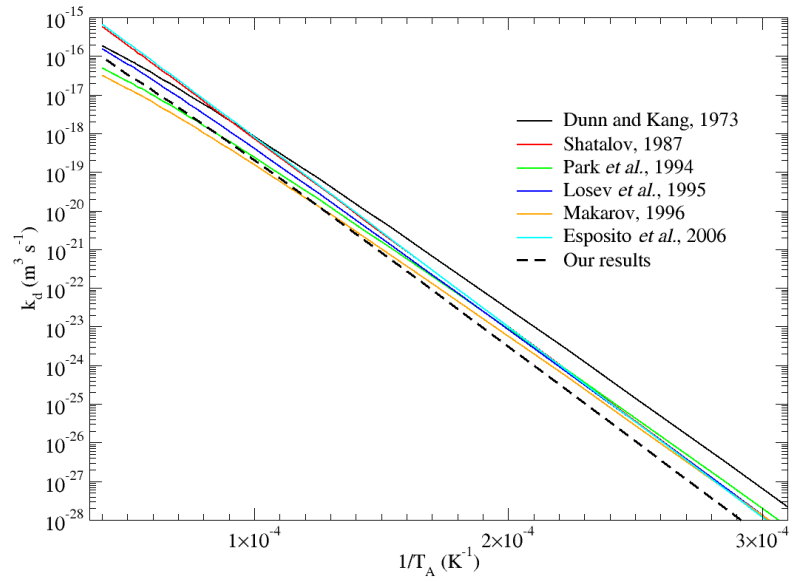

Fig. (5). Comparison between the present results and some reference data for the global rate coefficient of $\mathrm{N}_{2}$ dissociation under $\mathrm{N}$ impact as a function of $1 / T_{A}$.

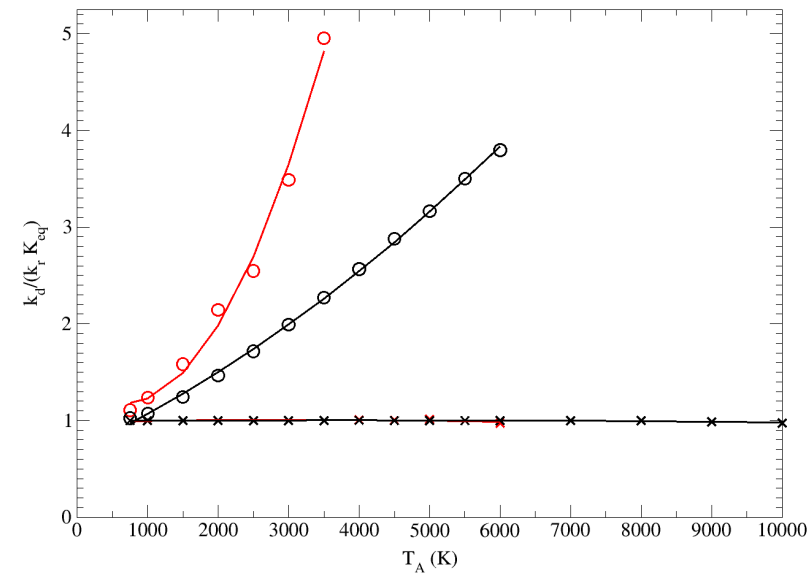

Fig. (6). Evolution with $T_{A}$ of the ratio $k_{f} /\left(k_{r} K_{e q}\right)$ for nitrogen (in black) and oxygen (in red) under atomic (crosses) or molecular (circles) impact. The lines correspond to polynomial interpolations of the second order.

\section{TWO-LEVEL DISTRIBUTION (TLD) MODEL}

In this section, an innovative reduced kinetic model is presented and firstly applied to pure $\mathrm{N}_{2}$ and $\mathrm{O}_{2}$ system [12, $44]$ and to $\mathrm{NO}$ formation in air in recombination conditions $[45,46]$. To build this model we have applied an inductive approach, starting from a detailed analysis of the STS time evolution of the vibrational distributions and gas composition in conditions similar to those met in different fluid dynamic systems. The basic assumptions are: whatever the initial distribution, the system reaches a quasi-steadystate (QSS) solution obtained as the balance of different processes; the QSS state obtained in the homogeneous calculations are encountered also in fluid dynamic conditions. Different behaviors have been observed [12] for the low energy and the high energy distribution. As a general behavior, the low energy distribution follows a Boltzmann function, while the distribution tail presents a plateau or a strong depletion respectively in recombination and dissociation regime. In recombination regime (see for example [4]) the global rates of endothermal chemical processes are influenced mainly by the distribution tail, also in presence of multi-quantum transitions, while the low energy distribution has a small influence, except close to equilibrium conditions. Therefore the Two-Level Distribution (TLD) model has been proposed, where the vibrational temperature is the parameter describing the low energy distribution, where anharmonic correction is negligible, and the population of the last vibrational level is representative of the distribution tail. Other quantities can be representative of the distribution tail, such as the total density of levels with $v>v_{\text {lim }}$, but it is more difficult to write the evolution equation for this quantity than for the last vibrational level.

To determine the dependence of the global rates on the relevant quantities, the evolution of the distribution relaxing from $T_{v}$ to $T$, with $T_{v}>T$ and with atomic density higher than equilibrium value (recombination regime) is calculated, using the state-to-state model, considering one chemical process at a time. Then, we correlate the population of the last level with the global rate of the process considered, even 
if multi-quantum transitions are considered. This approach gives the so called first order rates. In case of diatomic dissociation, the first order rates gives a good accuracy. In other cases, complex interplay between different processes makes the first order rates inaccurate and then higher order corrections are necessary. This is the case of NO formation where there is a strong influence of intermediate vibrational states of oxygen and nitrogen molecules, that cannot be accounted in the TLD model as it was developed in previous papers $[12,44]$.

\subsection{Model Description}

In this paper the TLD approach has been applied to a 5 species air mixture $\left(\mathrm{N}_{2}, \mathrm{~N}, \mathrm{O}_{2}, \mathrm{O}, \mathrm{NO}\right)$ and the kinetic processes included into the state-to-state model are $[12,44-46]$

- $\quad \mathrm{vTa}$ and $\mathrm{vTm}$ for $\mathrm{N}_{2}$ and $\mathrm{O}_{2}$

$\mathrm{N}_{2}(v)+\mathrm{N} \rightleftharpoons \mathrm{N}_{2}(v-\Delta v)+\mathrm{N}$

$\mathrm{N}_{2}(v)+\mathrm{N}_{2} \rightleftharpoons \mathrm{N}_{2}(v-1)+\mathrm{N}_{2}$

$\mathrm{O}_{2}(v)+\mathrm{O} \rightleftharpoons \mathrm{O}_{2}(v-\Delta v)+\mathrm{O}$

$\mathrm{O}_{2}(v)+\mathrm{O}_{2} \rightleftharpoons \mathrm{O}_{2}(v-1)+\mathrm{O}_{2}$

- $\quad \mathrm{vv}$ for $\mathrm{N}_{2}$ and $\mathrm{O}_{2}$

$\mathrm{N}_{2}(v)+\mathrm{N}_{2}(w-1) \rightleftharpoons \mathrm{N}_{2}(v-1)+\mathrm{N}_{2}(w)$

$\mathrm{O}_{2}(v)+\mathrm{O}_{2}(w-1) \rightleftharpoons \mathrm{O}_{2}(v-1)+\mathrm{O}_{2}(w)$

- dissociation by atoms and molecules

$\mathrm{N}_{2}(v)+N \rightleftharpoons 2 \mathrm{~N}+\mathrm{N}$

$\mathrm{N}_{2}(v)+N_{2} \rightleftharpoons 2 \mathrm{~N}+\mathrm{N}_{2}$

$\mathrm{O}_{2}(v)+\mathrm{O} \rightleftharpoons 2 \mathrm{O}+\mathrm{O}$

$\mathrm{O}_{2}(v)+\mathrm{O}_{2} \rightleftharpoons 2 \mathrm{O}+\mathrm{O}_{2}$

- $\quad$ first $\left(Z_{1}\right)$ and second $\left(Z_{2}\right)$ Zel'dovich reactions

$\mathrm{N}_{2}(v)+\mathrm{O} \rightleftharpoons \mathrm{NO}+\mathrm{N}$

$\mathrm{O}_{2}(v)+\mathrm{N} \rightleftharpoons \mathrm{NO}+\mathrm{O}$

The rates of vT and of dissociation by atoms are those calculated by Esposito [26, 27] and include multi-quantum transitions. The dissociation rates by molecules have been calculated by considering the same dependence on the vibrational quantum number as the dissociation by atoms, normalized to reproduce the thermal rates reported by Shalatov [47]. As a consequence, also the dissociation induced by molecular collisions includes multi-quantum transitions. For the first $\left(Z_{1}\right.$, see Eq. (33)) and second $\left(Z_{2}\right.$, see Eq. (34)) Zel'dovich reactions of NO formation, the rate coefficients have been taken from the work of Bose and Candler [48, 49]. The kinetic model is not complete because the mixed processes, as vT for $\mathrm{O}_{2}$ by $\mathrm{N}$ or $\mathrm{N}_{2}$, have been neglected, even if their contribution can be important. Moreover, the vibrational levels of NO have been neglected because the state-selective rates of NO formation consider only the ground state.
In the TLD approach, plasma composition is obtained by solving a system of macroscopic kinetic equations describing each species density and, for each molecular species, two other kinetic equations, one for the vibrational energy relaxation and one for the last vibrational level population. In this approach, chemical rates of dissociation are expressed as the sum of two terms: one as a function of gas and vibrational temperature, as in the MT approach, and the other as a function of gas temperature and of the last vibrational level population $\left(\alpha_{s}\right)$

$K_{S}^{d i s s}=K_{S}^{0}\left(T, T_{v, S}\right)+K_{S}^{1}\left(T, \alpha_{S}\right)$

where $K^{0}$ is the two-temperature rate used in MT models $[12,44,45]$ and $K_{S}^{1}\left(T, \alpha_{S}\right)$ is determined from the state-tostate calculations, with the requirement that it goes to zero when $\alpha_{s}$ approaches the $n_{S, v}^{B}\left(T_{v, S}\right)$. It should be emphasized that with Eq. (35) we also model processes involving states with $v<v_{\text {last }}$, but influenced mainly by highly excited vibrational states.

The dependence on $\alpha_{S}$ is the innovative point introduced by this model. In this way, chemical rates depend both on the low vibrational energy distribution through the vibrational temperature and on the high vibrational energy distribution through the last vibrational level population. The idea of the TLD model has been developed after accurate analysis of the vibrational distribution behavior [12, 44]. It has been observed [12, 44, 45], from STS calculations that, in recombination regime, the vibrational distribution reaches a quasi-stationary condition in a time interval of approximately $\mathrm{t}=10^{-8} \mathrm{~s}$. After that, dissociation rates shows a regular dependence on the population of the last vibrational level of the molecule involved into the process.

As an example, Fig. (7) shows the dependence of $\mathrm{N}_{2}+\mathrm{N}$ global dissociation rates as a function of last level population obtained from STS time dependence calculations at constant pressure for different gas temperature $\left(T_{g}=1,000 \mathrm{~K}, 2,000 \mathrm{~K}\right.$, $5,000 \mathrm{~K}$ [44]. These calculations have been performed by considering the pure gas $\mathrm{N}_{2} / \mathrm{N}$ and choosing an initial condition in which recombination should dominate the kinetics, i.e. initial vibrational temperature of $\mathrm{N}_{2}$ greater than gas temperature $\left(T_{v 0}=8,000 K>T_{g}\right)$ and initial gas composition with molar fractions $\chi_{\mathrm{N}}=0.9$ and $\chi_{\mathrm{N}_{2}}=0.1$.

As observed, $\mathrm{N}_{2}+\mathrm{N}$ dissociation rate shows a linear dependence from last level population, with the only exception of short time behavior corresponding to the time interval necessary to reach quasi-steady state conditions.

For the relaxation of the vibrational energy of the s-th molecular specie, the following equation is used

$\frac{d E_{v, S}}{d t}=G_{v T, S}\left(T, T_{v, S}\right)-L_{v T, S}\left(T, T_{v, S}\right)$

in which the gain (G) and the losses (L) are due to vT processes and have been calculated summing up the state-tostate over a Boltzmann vibrational distribution multiplied by 
the variation of internal energy in the given transition. This strategy is different from MT model approach in which the relaxation depends on $p \tau$ values [13-15]. In any case, the internal energy is related to the vibrational distributions considering the anharmonic energy levels of the diatomic species [26, 27]. It must be pointed out that in the TLD the contribution of the vibrational energy to the chemical kinetics is very weak, except when the system approaches the chemical equilibrium.

The master equation of the last vibrational level population $\left(\alpha_{s}\right)$ of the s-th molecular specie is obtained by considering gains and losses coming from chemical processes of dissociation by vTa and vTm, NO formation reactions (first second Zel'dovich reaction $Z_{1}$ in the case of $\mathrm{N}_{2}$ and second Zel'dovich reaction $Z_{2}$ in the case of $\mathrm{O}_{2}$ ), and only losses due to $\mathrm{vTa}$ and $\mathrm{vTm}$ processes towards lower level $\left(v<v_{\text {last }}\right)$, since, in recombination regime [12, 44], the $\mathrm{vv}$ and the vT up-pumping can be considered negligible.

$$
\begin{aligned}
& \frac{d \alpha_{S}}{d t}=G_{S}-L_{S} \alpha_{S}=K^{r_{-} \vee T a} N^{3}+K^{r_{-} \vee T T m} N^{2} N_{2} \\
& +K^{r_{-} Z_{i}} N O N-\left(K^{d_{-} \vee T a} N\right. \\
& \left.+K^{d_{-} v T m} N_{2}+K^{d_{-} Z_{i}} O+K_{v_{\text {last }} \rightarrow v}^{v T a} N+K_{v_{\text {last }} \rightarrow T \text { t }}^{v T m} N_{2}\right)
\end{aligned}
$$

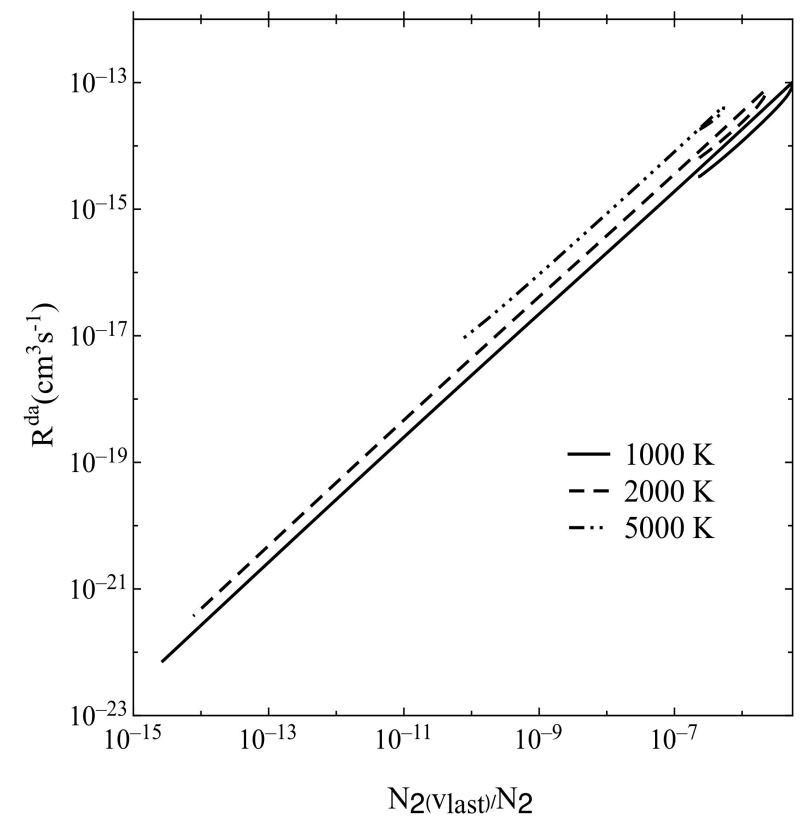

Fig. (7). $N_{2}+\mathrm{N}$ dissociation rates calculated from state-to-state kinetic as a function of the last level population of $\mathrm{N}_{2}$ ( $\alpha=$ $\left.\mathrm{N}_{2}\left(v_{\text {last }}\right) / \mathrm{N}_{2}\right)$ for initial conditions $T_{v 0}=8000 \mathrm{~K}$ and $\chi_{\mathrm{N}}=0.9$.

\subsection{Results}

In this section, results of the implementation of TLD model and the comparison with STS and MT model are presented. Fig. (8) shows the time evolution of molar fractions of (a) $\mathrm{N}_{2}$ and (b) $\mathrm{O}_{2}$ calculated with the three models in the condition in which an air mixture is relaxing at atmospheric pressure to $\mathrm{T}=1000 \mathrm{~K}$, starting with initial vibrational temperatures $T_{v, N_{2}}=T_{v, O_{2}}=10,000 \mathrm{~K}$ and with the following initial molar fractions: $\mathrm{N}_{2}=810^{-4}, \mathrm{~N}=$ $0.7992, \mathrm{O}_{2}=210^{-4}, \mathrm{~N}=0.1998, \mathrm{~N} \mathrm{O}=0[45,46]$. As it can be seen, the TLD model determines molar fractions of $\mathrm{N}_{2}$ and $\mathrm{O}_{2}$ molecules in a very good agreement with STS in all the time interval while MT agrees with the other models only for $t>10^{-5} \mathrm{~s}$. In this case, the MT model underestimates the contribution of the overpopulated distribution tails to the chemical processes. As a consequence, for $\mathrm{N}_{2}$ this behavior results in lower dissociation rates, speeding up the recombination kinetics. The same behavior is observed for $\mathrm{O}_{2}$, where MT does not detect the decreasing of the molar fraction starting around $t=10^{-8} \mathrm{~s}$, because MT model does not consider the contribution of the last level and, as for nitrogen, anticipates the oxygen recombination with respect to STS. To calculate the time evolution of NO molecules, as a first attempt, the same approach of the TLD model used for pure $\mathrm{N}_{2} / \mathrm{N}$ and $\mathrm{O}_{2} / \mathrm{O}$ has been applied also to the two Zel'dovich reactions $[45,46]$. Thus, to determine the dependence of the rate of the first and second Zel'dovich reactions on the last vibrational level of $\mathrm{N}_{2}$ and $\mathrm{O}_{2}$, respectively, STS calculations have been performed in recombination regime by considering both the kinetics of pure $\mathrm{N}_{2} / \mathrm{N}$ and $\mathrm{O}_{2} / \mathrm{O}$ mixture, but adding only the process under study, i.e. $\mathrm{N}_{2}+\mathrm{O}$, in the first case and $\mathrm{O}_{2}+\mathrm{N}$, in the second one. In this way, the two corresponding rate coefficients were written as a function of the last vibrational level of $\mathrm{N}_{2}$ for the $\mathrm{N}_{2}+\mathrm{O}$ process and of $\mathrm{O}_{2}$ for the $\mathrm{O}_{2}+\mathrm{N}$ process (see Eq. (35)). Unfortunately, the corresponding TLD results showed a bad reproduction of STS results for O, $\mathrm{NO}$ and $\mathrm{N}$ molar fractions for $t>10^{-4} \mathrm{~s}[45,46]$.

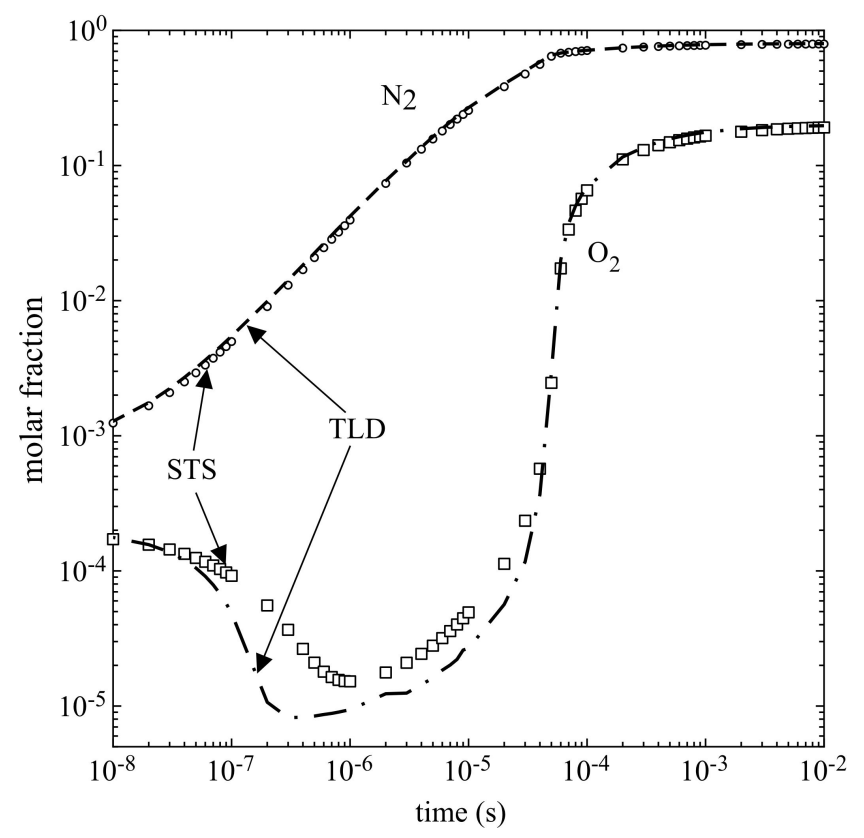

Fig. (8). Molar fraction time evolution of $\mathrm{N}_{2}$ and $\mathrm{O}_{2}$ for STS and TLD kinetic models in recombination regime. 


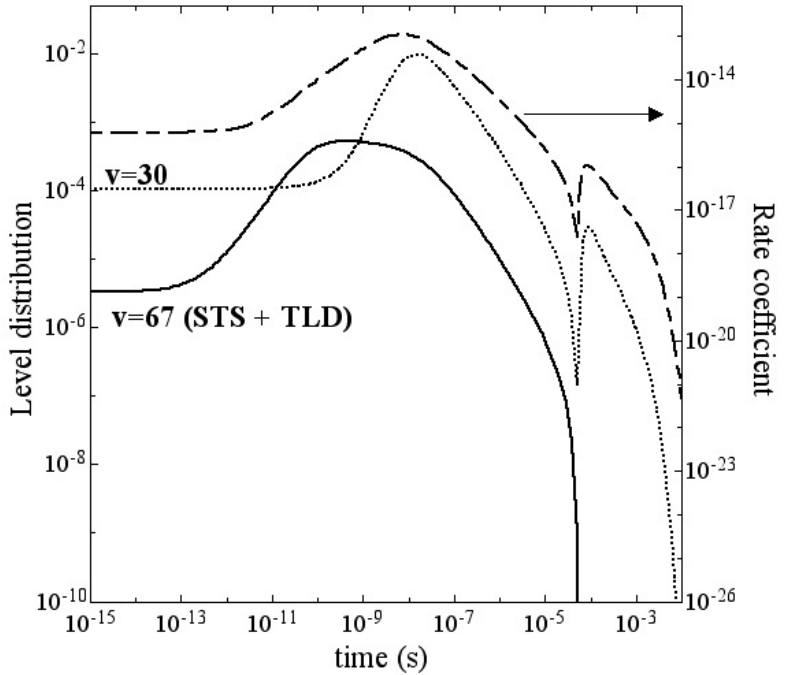

Fig. (9). Time evolution of $\mathrm{N}_{2}(\mathrm{v}=67)$ and $\mathrm{N}_{2}(\mathrm{v}=30)$ vibrational level distribution and of the first Zel'dovich rate coefficient (right axis) calculated in the complete STS model, in recombination regime.

The reason of this failure can be found by observing that a strong and complex correlation between the two Zel'dovich reactions occurs. Thus, the dependence of the rate should be analyzed by including the complete kinetics in the calculations. Fig. (9) reproduces the time evolution of $\mathrm{N}_{2}+\mathrm{O}$ rate coefficient (right axis) and of the $\mathrm{N}_{2}(\mathrm{v}=30)$ and $\mathrm{N}_{2}$ $(\mathrm{v}=67)$ level population (one intermediate and the last vibrational level of $\mathrm{N}_{2}$, respectively) obtained by performing complete kinetics STS calculations. As it can be seen, $\mathrm{N}_{2}+\mathrm{O}$ rate coefficient looses the correlation with the last vibrational level and seems to be influenced by the time evolution of the intermediate part of the level distribution population $(\mathrm{v}=30)$. The second Zel'dovich reaction $\mathrm{O}_{2}+\mathrm{N}$ strongly affects the vibrational distribution of $\mathrm{N}_{2}$, even if it is not directly involved in the process by creating a channel which produces $\mathrm{N}_{2}$ molecules in intermediate levels. This channel is created by the following chain processes between the two Zel'dovich reactions

$$
\begin{aligned}
\mathrm{O}_{2}+\mathrm{N} \rightarrow \quad \mathrm{NO} & +\mathrm{O} \\
\downarrow & \\
\mathrm{NO} & +\mathrm{N} \rightarrow \mathrm{N}_{2}\left(v_{\text {med }}\right)+\mathrm{O} \\
\mathrm{O}+\mathrm{NO} \rightarrow & \mathrm{N}+\mathrm{O}_{2} \\
\downarrow & \\
& \\
& +\mathrm{NO} \rightarrow \mathrm{N}_{2}\left(v_{\text {med }}\right)+\mathrm{O}
\end{aligned}
$$

These chain reactions are the preferential channel to $\mathrm{N}_{2}$ formation, reducing the contribution of $\mathrm{N}+\mathrm{N}$ direct recombination in high energy states and resulting in the depletion of the tail of the vibrational distribution of $\mathrm{N}_{2}$ [50]. In this case the NO formation comes mainly from intermediate levels of $\mathrm{N}_{2}$. To appreciate this behavior, we have to consider the full state-to-state kinetics and we have to add a second order correction $K^{2}$. To take into account the dependence of the rate of the first Zel'dovich reaction on intermediate level population, the second order correction should be constructed considering the channels in Eqs. (38) and (39) and therefore it should depend on the reactant of the reactions, i.e. $\mathrm{N}, \mathrm{N}_{2}, \mathrm{NO}$ and $\mathrm{O}$ and thus be proportional to the population of intermediate state $N_{2}\left(v_{m}\right)$ :

$$
K_{\mathrm{N}_{2}+\mathrm{O} \rightarrow \mathrm{NO}+\mathrm{O}}=K^{T L D}\left(T, \alpha_{\mathrm{N}_{2}}\right)+K^{2}\left(T, \mathrm{~N}, \mathrm{~N}_{2}, \mathrm{NO}, \mathrm{O}\right)
$$
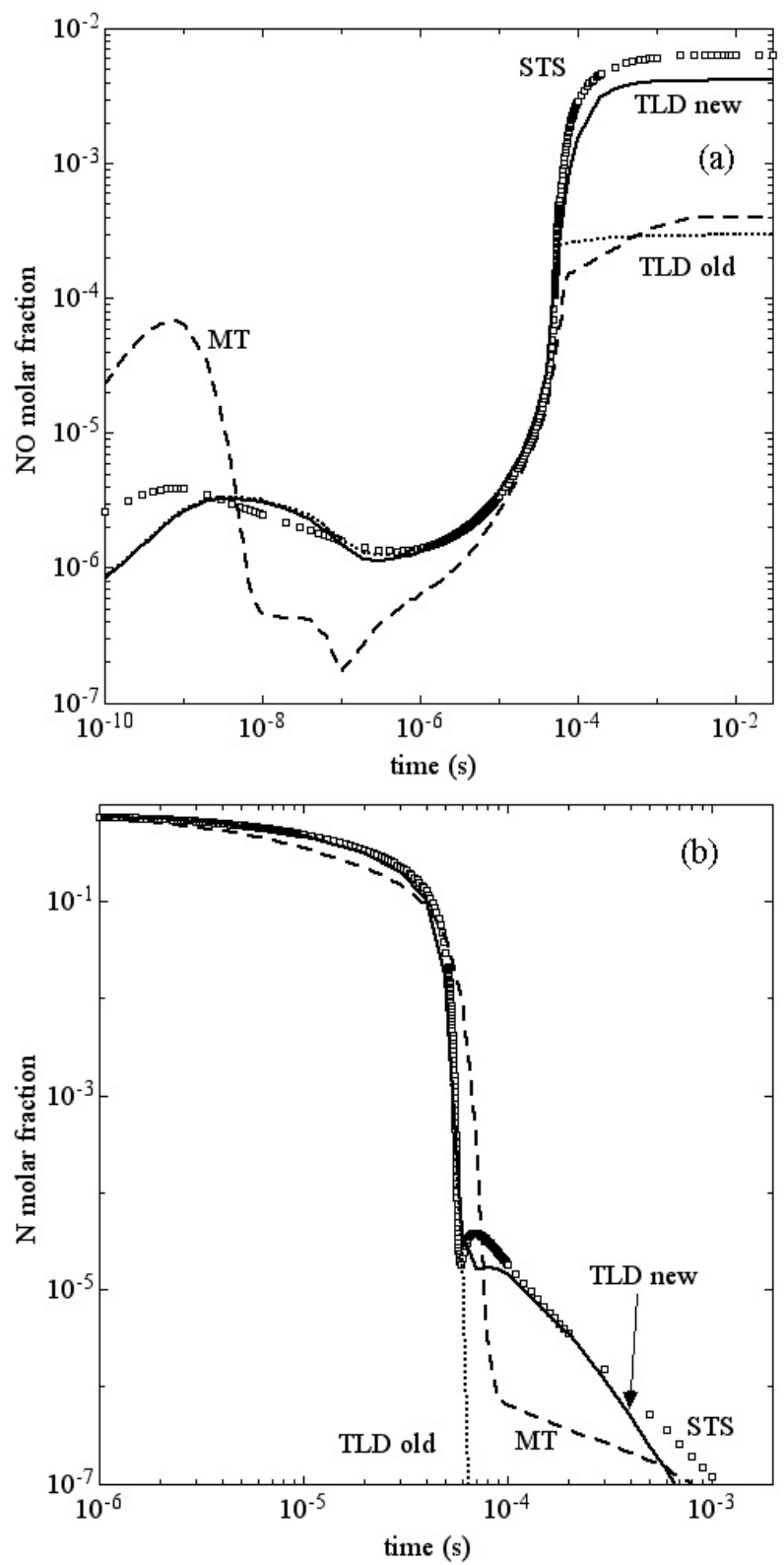

Fig. (10). Comparison between STS, MT and new and old TLD results obtained for (a) $\mathrm{NO}$ and (b) $\mathrm{N}$ molar fraction time evolution is shown. The old TLD corresponds to $\mathrm{N}_{2}+\mathrm{O}$ rate fitting performed using Eq. (35), while the new one using Eq. (40).

New TLD results obtained by applying eq. (40) for $\mathrm{N}_{2}+\mathrm{O}$ rate coefficient are shown in Fig. (10) where comparison between STS, MT and new and old TLD results obtained for (a) $\mathrm{NO}$ and (b) $\mathrm{N}$ molar fraction time evolution is shown. The old TLD corresponds to $\mathrm{N}_{2}+\mathrm{O}$ rate fitting performed by 
using Eq. (35). As it can be observed, new approach of rate fitting gives much more better agreement with STS, respect to old TLD. Again, we want to stress that the state-to-state model used to calculate the results presented in this section includes multiquantum transitions for VT processes with atoms and for dissociation by both atoms and molecules and includes also $\mathrm{VV}$ processes.

\section{MULTI-INTERNAL-TEMPERATURE MODELS}

In classical multitemperature models, a unique vibrational temperature is used to describe the vibrational distribution function. In this section, we propose to use several internal vibrational temperatures to better represent the vibrational nonequilibrium of a molecular flow. In the macroscopic model obtained, energy exchange terms and macroscopic rate coefficients are derived self-consistently from a state to state database $[51,52]$. Recently, this model was successfully applied to FIRE 2 flight conditions where relaxation and dissociation processes are relatively fast due to a very high translational temperature [51]. In this work, we apply this model to a low earth orbit return condition where the translational temperature is lower and nonequilibrium processes dominate in the entire shock layer.

\subsection{Model Description}

In this work we consider a vibrational state to state model for $N_{2}$, using the $v_{\max }=61$ vibrational energy levels of Leroy [53]. The reaction rate constants for multiquanta $V T$ transitions are computed by means of the Forced Harmonic Oscillator (FHO) model, as used by Adamovich et al. [54]. The state dependent dissociation rate constants are also estimated thanks to the FHO model. As demonstrated by Adamovich et al. [55], VV processes have only a little effect on the dissociation behind a shock wave in $N_{2}$ and then we have neglected them in this work. We then performed the simulation of relaxation of the flow behind a shock wave. The reactive Euler equations are solved in an ODE form, using the Rankine-Hugoniot relations to compute the initial condition just behind the shock. The simulation is performed for conditions derived from the Hermes $\mathrm{H} 1$ test case, given in Table 2.

Table 2. Free stream and post shock conditions used.

\begin{tabular}{|c|c|c|c|}
\hline & Velocity $\left(\boldsymbol{m s}^{-\mathbf{1}}\right)$ & Pressure (Pa) & Temperature $(\boldsymbol{K})$ \\
\hline \hline Free stream & 7181 & 2.5 & 205.3 \\
\hline Post shock & 1206 & 1762 & 24324 \\
\hline
\end{tabular}

For all computations, an effective vibrational temperature $T_{V}$ is extracted from the energy of the vibrational distribution function (VDF), according to:

$$
\frac{\sum_{v=0}^{v_{\max -1}} e^{-\frac{E_{v}}{k_{B} T_{V}}} \cdot e_{v}}{\sum_{v=0}^{v_{\max -1}} e^{-\frac{E_{v}}{k_{B} T_{V}}}}=\frac{\sum_{v=0}^{v_{\max -1}} N_{2}^{v} \cdot e_{v}}{\sum_{v=0}^{v_{\max -1}} N_{2}^{v}}
$$

where $e_{v}$ is the vibrational energy of the level $\mathrm{v}$ per unit mass, $E_{v}$ is the energy of the vibrational level $\mathrm{v}$ in $\mathrm{J}$ and $k_{B}$ is the Boltzmann constant.

Fig. (11a) shows that $T_{V}$ quickly increases as a consequence of the $V T$ processes and reaches a maximum $4 \mathrm{~mm}$ after the shock where the dissociation processes are more efficient than VT processes and then deplete the vibrational energy. This distance depends on the test-case but corresponds to the zone of incubation of the vibrational energy behind a shock wave [16]. Then $T_{V}$ decreases and equilibrates with the translational temperature $\mathrm{T}$, without overshooting it because of the depletion of vibrational energy by dissociation. Fig. (11b) shows the chemical dynamics which starts slowly in the incubation zone, where only the low-lying levels are populated. Dissociation becomes fast at $x=4 \mathrm{~mm}$ where the high lying vibrational levels are populated. The dissociation phenomenon is slow: after $10 \mathrm{~cm}$, which is the typical thickness of the shock layer, $\mathrm{N}_{2}$ is still dissociating. As dissociation processes are endothermic, Fig. (11a) shows that the temperatures decrease as the flow dissociates.

In this work, to take into account the vibrational nonequilibrium we choose to model the vibrational distribution function (VDF) by several groups of levels, within which the levels are assumed to follow a Boltzmann distribution at the temperature of the group $T_{V}^{i}$. Hence, each group $i$ is described by its density $\rho_{N_{2}^{i}}$ (and the corresponding mass fraction $y_{N_{2}^{i}}$ ) and its vibrational temperature $T_{V}^{i}$ (and the corresponding vibrational energy $\left.e_{V i b}^{i}\right)$. This leads to a continuity equation and an energy equation for each group:

$$
\begin{aligned}
& \partial_{t}\left(\rho_{N_{2}^{i}}\right)+\partial_{x}\left(\rho_{N_{2}^{i}} u\right)=\omega_{\text {diss rec }}^{i}+\omega_{V T}^{i} \\
& \partial_{t}\left(\rho \cdot y_{N_{2}^{i}} \cdot e_{V i b}^{i}\left(T_{V}^{i}\right)\right)+\partial_{x}\left(\rho u \cdot y_{N_{2}^{i}} \cdot e_{V i b}^{i}\left(T_{V}^{i}\right)\right)=\Omega_{C V}^{i}+\Omega_{V T}^{i}
\end{aligned}
$$

where $u$ is the velocity of the flow and $\rho$ is the density. $\omega_{\text {diss rec }}^{i}$, resp. $\omega_{V T}^{i}$ are the chemical source terms resulting from dissociation and recombination, respectively from $V T$ excitation. $\Omega_{C V}^{i}$, resp. $\Omega_{V T}^{i}$ are the vibrational energy source terms resulting from dissociation and recombination, respectively from $V T$ excitation. These equations are derived from the equations of continuity for the vibrational levels. For example, the rate of dissociation and recombination for the group $i$ is obtained by summing the continuity equations over the vibrational levels $v$ in group $\mathrm{i}$ :

$$
\omega_{\text {diss rec }}^{i}=m_{N_{2}} \sum_{M=N, N_{2}} \sum_{v \in i}\left(k_{v}^{d, M} \cdot N_{2}^{v} \cdot M-k_{v}^{r, M} \cdot N^{2} \cdot M\right)
$$

where $m_{N_{2}}$ is the molar mass of $N_{2}, k_{v}^{d, M}$ and $k_{v}^{r, M}$ are the dissociation and recombination rate constants for the level $v$ known from the database. $N_{2}^{v}$ and $\mathrm{N}$ are the molar density of the corresponding species. 


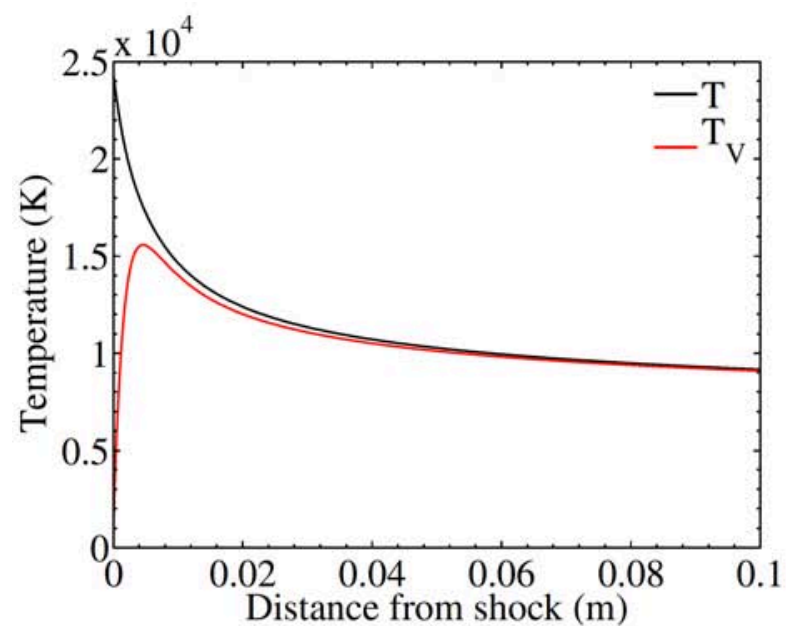

(a) Evolution of temperatures $T$ and $T_{V}$

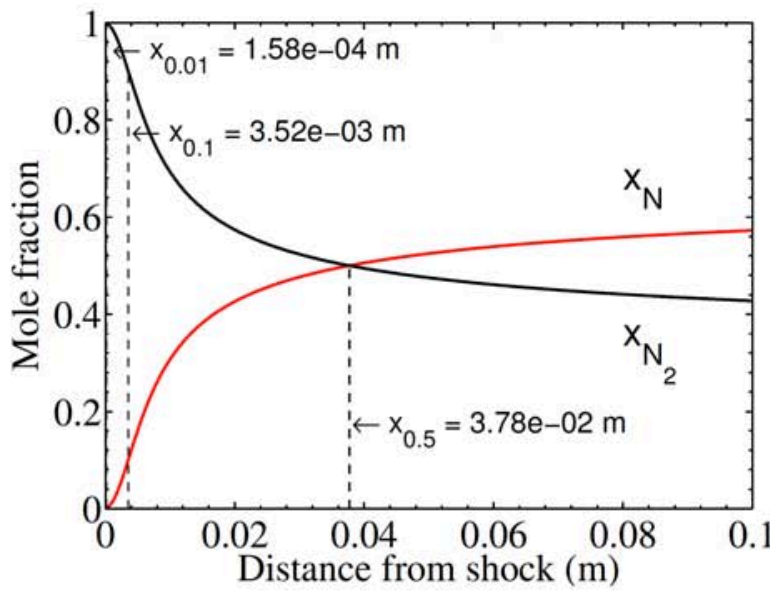

(b) Evolution of composition

Fig. (11). Flowfield behind the shock wave computed with the vibrational collisional model for the conditions given in Table 2.

Assuming that the vibrational distribution within a group is a Boltzmann distribution at $T_{V}^{i}$, we get

$\omega_{\text {diss/rec }}^{i}=m_{N_{2}} \sum_{M=N, N_{2}}\left[\sum_{v \in i} k_{v}^{d, M} \cdot f_{i}\left(v, T_{V}^{i}\right) \cdot N_{2}^{i} \cdot M-\sum_{v \in i} k_{v}^{r, M} \cdot N^{2} \cdot M\right]$

and then the rate of dissociation and recombination for the group $i$ is a function of $T, T_{V}^{i}$ and the densities $N_{2}^{i}$ and of $N_{2}$ and $\mathrm{N}$. The other source terms can be derived selfconsistently using the same procedure [52].

\subsection{Results}

In this section, the reduced model is applied on the $\mathrm{H} 1$ test case given in Table $\mathbf{2}$ with one group to assess the Boltzmann assumption. Then it is applied with two groups to take into account the different behavior of low lying and high lying levels. The respective results are compared with the detailed vibrational model.

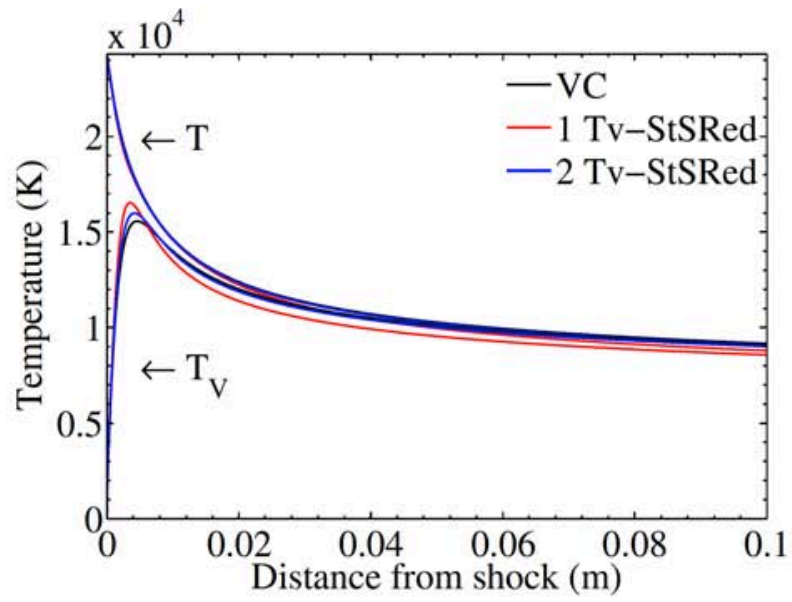

(a) Evolution of temperatures $T$ and $T_{V}$
Fig. (12a) shows that both the model with one group (in red) and with two groups (in blue) predict a vibrational temperature $T_{V}$ that reaches a maximum value at the same location, then decreases without overshooting T. However the relaxation predicted by the one group model is too fast, which leads to a temperature too low by several hundreds of Kelvin at a distance representative of the shock layer thickness $(10 \mathrm{~cm})$. The two group model (in blue) overlaps the detailed model (in black), so the vibrational energy is well predicted. The one group model slightly overestimates the dissociation rate as shown in Fig. (12b). Though small, this difference explains the more rapid cooling of $T$ and $T_{V}$, due to the conversion of translational and vibrational energy into formation energy. We also note a slowing of equilibration between the translational and the vibrational modes. The two-group model (in blue) reproduces the results of the detailed collisional model with a very good agreement.

These results indicate that nonequilibrium effects affect the flow. To investigate these nonequilibrium effects on the

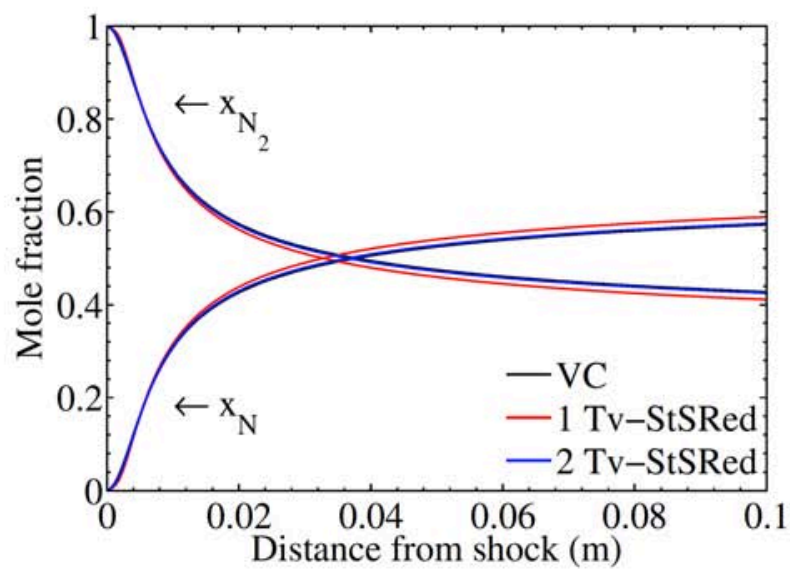

(b) Evolution of composition

Fig. (12). Flowfield behind the shock wave computed with the detailed collisional model and with the reduced model with 1 and with 2 groups for the conditions given in Table $\mathbf{2}$. 


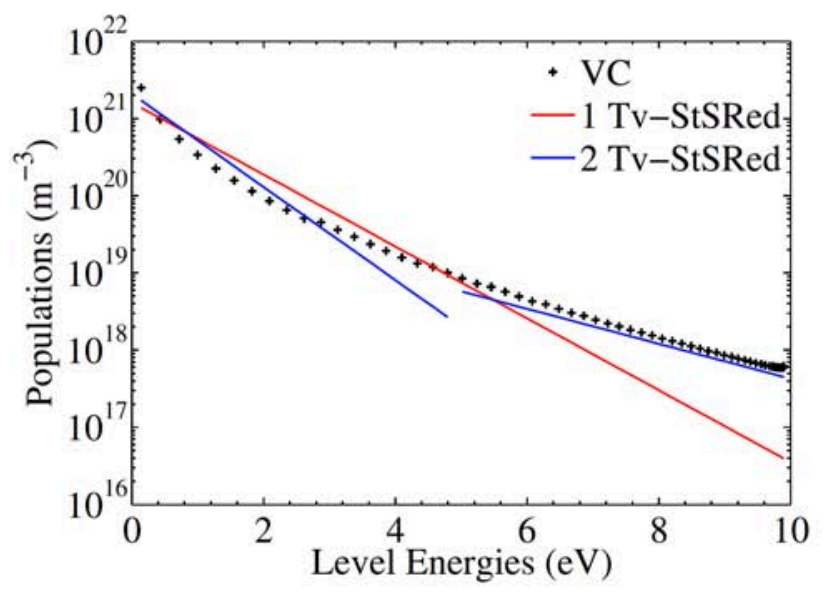

(a) $x=x_{0.01}=1.58 \times 10^{-4} \mathrm{~m}$

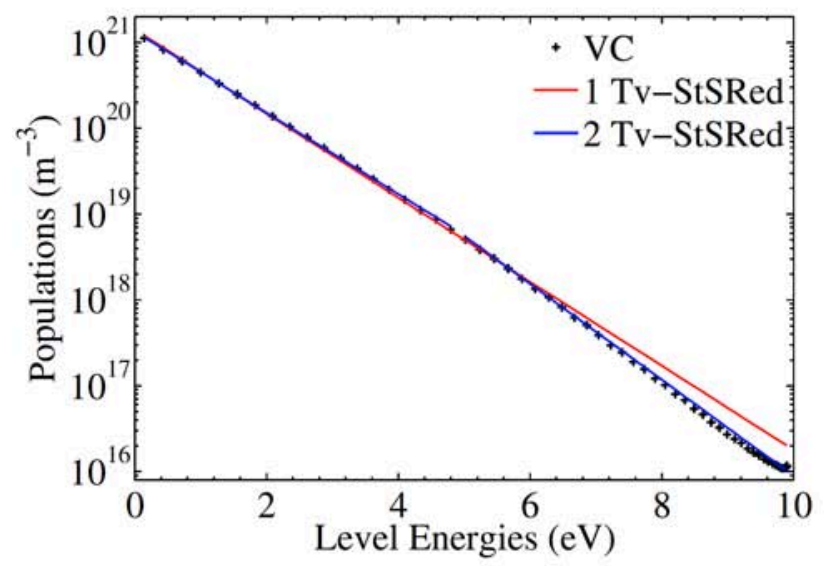

(c) $x=x_{0.5}=3.78 \times 10^{-2} \mathrm{~m}$

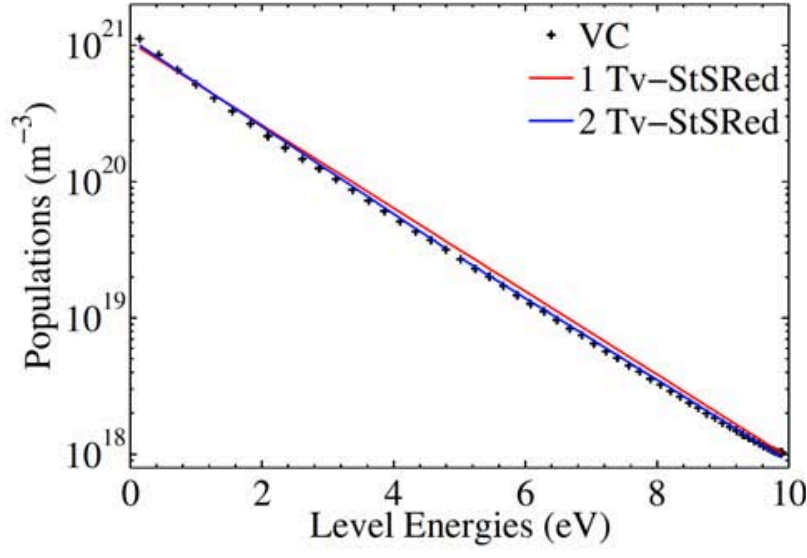

(b) $x=x_{0.1}=3.52 \times 10^{-3} \mathrm{~m}$

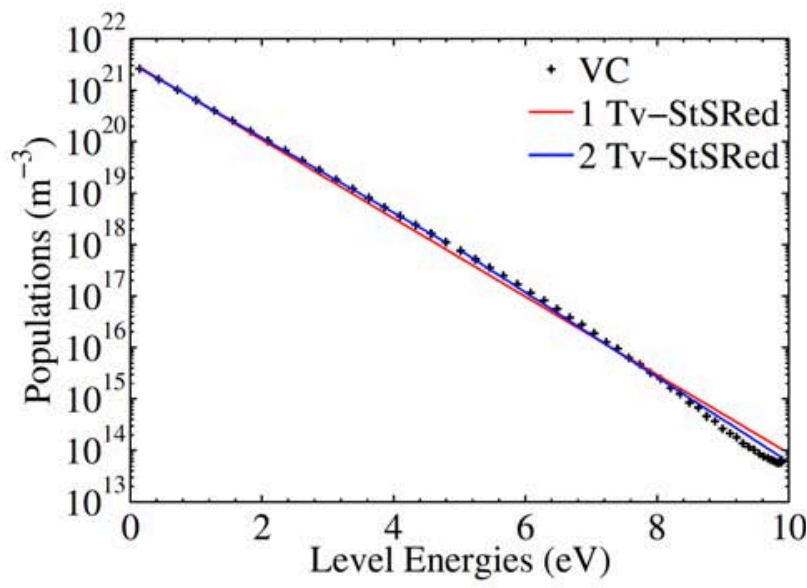

(d) $x=1 \mathrm{~m}$

Fig. (13). VDF computed with the detailed vibrational collisional model and the 1 group and 2 groups models.

coupling between vibrational excitation and kinetics, we plot the VDF at several significant locations represented on Fig. (11b):

- $x=x_{0.01}$ where the mass fraction $x_{N}=0.01$; in the incubation zone.

- $x=x_{0.1}$ where the mass fraction $x_{N}=0.1$; where dissociation starts to be significant.

- $x=x_{0.5}$ where the mass fraction $x_{N}=0.5$; where half dissociation is achieved.

- $\quad x=1 \mathrm{~m}$; at the end of the simulation domain.

Fig. (13a) shows the rapid vibrational excitation that happens just after the shock and populates the high levels. At this location there is a significant nonequilibrium and a 2 group model is required to accurately model it. It can be observed that the vibrational temperature based on the vibrational energy gives a much better representation of the VDF than the one obtained using a vibrational temperature based on the population of the two first vibrational levels. When dissociation begins to be significant (Fig. 13b) the VDF is almost Boltzmann. But after that, as half of the dissociation is achieved, we observe a depletion of high vibrational levels on Fig. (13c). The translational temperature $\mathrm{T}$ has decreased below $15,000 \mathrm{~K}$ so that dissociation happens from higher vibrational levels. This leads to a depletion of the high lying levels that are populated by $V T$ processes and depopulated by dissociation. This depletion remains up to $1 \mathrm{~m}$ after the shock where equilibrium is still not reached as shown on Fig. (13d).

Except in the incubation zone just after the shock front, the one group model is quite close to the VDF computed with the state-to-state model. However this approximation is obviously not sufficient here; as seen in Fig. (12b) the flow predicted by the one-group model $10 \mathrm{~cm}$ after the shock is too much dissociated, and correspondingly the temperatures are too low. Conversely, the two-group model is able to predict dissociation and thus gives the right evolution of temperatures. This is because it allows to predict more accurately the evolution of the high lying levels (Fig. 13c) than the one-group model.

To explain the difference between the two models, we investigate the source terms. The first source term that influences the vibrational temperature is $\Omega_{V T}$. Concerning 


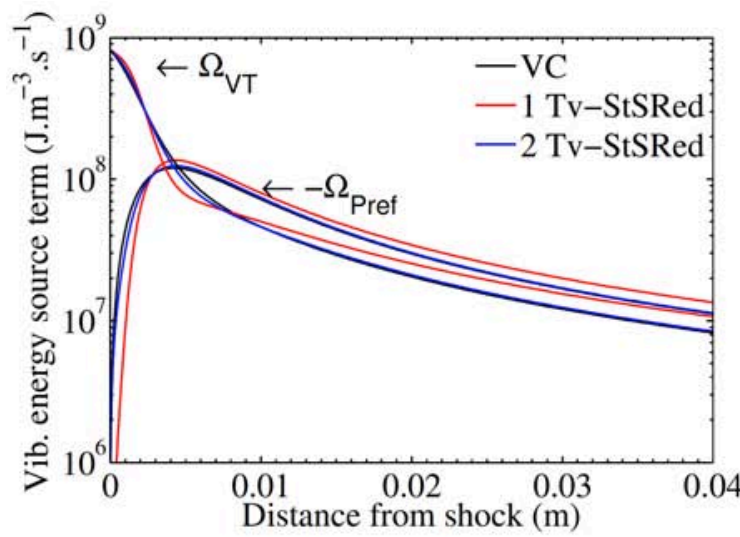

(a)

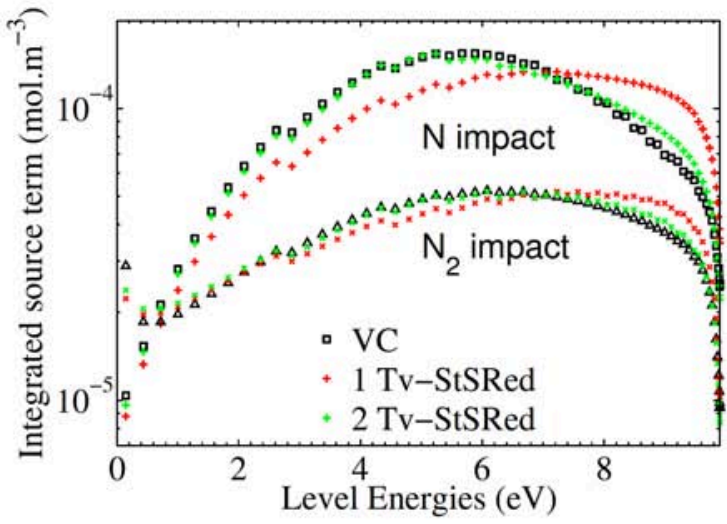

(b)

Fig. (14). (a) Post-shock evolution of sources terms of vibrational energy $\Omega_{V T}$ and $\Omega_{\text {Pref }}$ and (b) Contribution of each level to the dissociation process over the whole simulation time.

the coupling with chemistry, a molecule that dissociates will change the vibrational temperature only if its energy is different from the average vibrational energy. Thus the relevant source term is not $\Omega_{C V}$ but $\Omega_{\text {Pref }}$, defined as:

$\Omega_{\text {Pref }}=\Omega_{C V}-\omega_{N_{2}} \cdot e_{V i b}^{N_{2}}$

where $\Omega_{C V}$ is the total chemistry-vibration source term for $N_{2}, \omega_{N_{2}}$ the total chemical source term and $e_{V i b}^{N_{2}}$ is the average vibrational energy of $N_{2}$.

Fig. (14a) shows these two source terms as a function of the distance from the shock for the detailed vibrational model and the 1 group and 2 group reduced model. We note that the reduced model with one group qualitatively reproduces the behavior of both source terms. However, a two-group model is needed to have a correct prediction of both source terms. On Fig. (14b) is plotted the contribution to dissociation of each vibrational level $I^{v}$, integrated over the whole simulation time as follows:

$I^{v}=\int_{t=0}^{t_{f}}\left(k_{d}^{M, v} \cdot N_{2}^{v}-k_{r}^{M, v} \cdot N^{2}\right) \cdot M \cdot d t$

Fig. (14b) shows that the one-group model overestimates the dissociation from high-lying levels. First this induces in an overprediction of the dissociation rate, resulting in a decrease of the temperatures. Secondly, this induces an overestimation of the vibrational energy lost during dissociation, which decreases $T_{V}$. By improving the representation of these high levels, the two-group model better describes the level-dependent dissociation, which eventually leads to a better prediction of the nonequilibrium flow.

Finally, the $\mathrm{H} 1$ test case studied in this section presents a different behavior from the FIRE 2 test case investigated in [51]; the high-lying levels are depleted slowly contrary to the FIRE 2 test case where the VDF quickly reaches Boltzmann equilibrium. However, the reduced model with two temperatures is able to predict the VDF, and then allows to predict the nonequilibrium of the flow for the $\mathrm{H} 1$ test-case.

\section{COARSE-GRAIN MODELS}

The most detailed databases developed in the literature are rovibrational databases [22-25]. In [58], a 0D rovibrational collisional model has been developed for the 9390 rovibrational energy levels of the electronic ground state of the nitrogen molecule with the reaction rate coefficients of the ab initio database developed at NASA Ames Research Center for the $\mathrm{N}_{2}+\mathrm{N}$ system [22, 23]. Then different reduced models have been developed based on this rovibrational database. In particular, as an alternative to vibrational-collisional models, in [10], the 9390 rovibrational energy levels of $\mathrm{N}_{2}$ have been lumped into a smaller number of bins as a function of the total internal energy, independently of their vibrational and rotational contributions. This approach is more general since the assumption of rotational equilibrium can be relaxed. The rate coefficients are averaged for each bin based on a uniform distribution of the energy levels within a bin. The Master equation for the reduced mechanism has been coupled with a one-dimensional flow solver to study excitation and dissociation processes behind a strong shock wave in a nitrogen flow for conditions expected for reentry into Earth's atmosphere. For the conditions of Table 2, Figs. $(\mathbf{1 5}, \mathbf{1 6})$ show the influence of the number of bins on the evolution of the composition and of the translational and internal temperatures behind the shock. The solid line corresponds to the solution for 200 bins which is very close to the reference solution with the detailed rovibrational model.

We point out that a rovibrational collisional coarse-grain model based on a uniform distribution of the internal energy levels into bins allows to describe accurately the internal energy relaxation and dissociation processes based on a reduced number of equations (of the order of 50). Furthermore, in [10], it is shown that the induction times for thermal relaxation and dissociation with a coarse-grain model are larger than the ones obtained by means of vibrational collisional and multitemperature models assuming thermal equilibrium between the rotational and translational energy modes. 


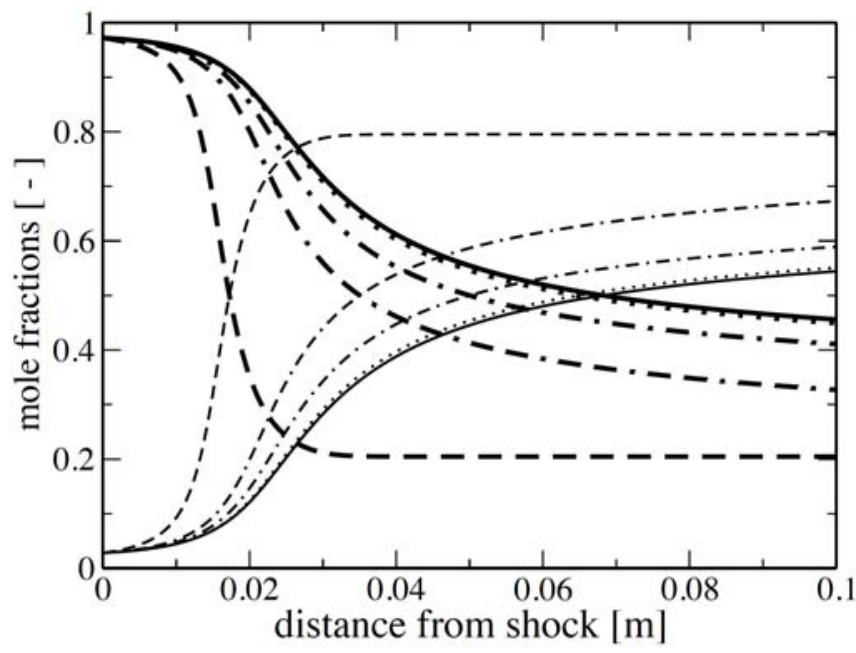

Fig. (15). Post-shock nitrogen atom mole fraction (thin lines) and nitrogen molecule mole fraction (thick lines) as a function of the distance from the shock based on the post-shock nonequilibrium conditions given in Table 2. Uniform Rovibrational Collisional (URVC) coarse-grain model with different numbers of bins (-- $5, \cdot--\cdot 10, \cdot-\cdot 20, \ldots 50,-200$ bins).

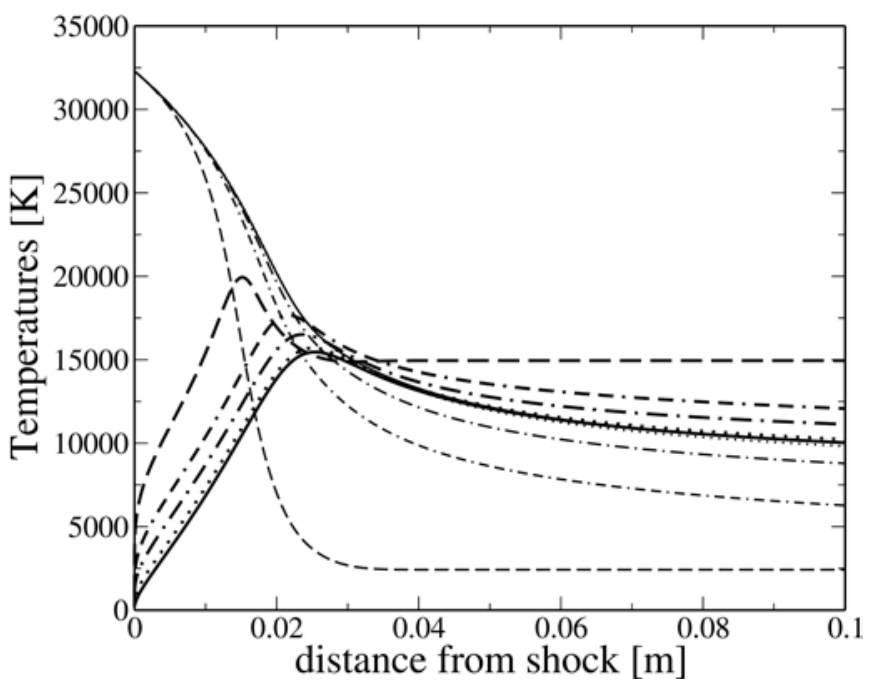

Fig. (16). Post-shock translational temperature (thin lines) and internal temperature (thick lines) as a function of the distance from the shock based on the post-shock nonequilibrium conditions given in Table 2. Uniform Rovibrational Collisional (URVC) coarse-grain model with different numbers of bins $(---5, \cdot--\cdot 10, \cdot-\cdot 20$, ... 50, - 200 bins).

\section{CONCLUSION}

In the present work we have presented the derivation of different types of macroscopic models by increasing order of complexity for the vibration-chemistry coupling in nonequilibrium flows for re-entry applications.

First, using an approach based on nonequilibrium thermodynamics, we have determined the global rate coefficients of (a) dissociation of $\mathrm{N}_{2}$ and $\mathrm{O}_{2}$ under parent molecular or atomic impact and (b) backward molecular recombination. Although the elementary forward and backward rate coefficients have a ratio equal to the corresponding equilibrium constant, this property is not observed for global rate coefficients unless the departure from equilibrium is weak. We have demonstrated this basic property which results from the nonequilibrium distribution of excited states during a global process. Such a study has also been performed for (c) ionization $[n=1, \alpha=1, \beta=1$ in (2)] of $X \equiv \mathrm{C}, \mathrm{N}, \mathrm{O}$, and Ar under $P \equiv e^{-}$impact and (d) ionic three-body recombination (see [57]). Then we have presented a Two-Level Distribution (TLD) model, in which a relaxation equation for vibrational temperature is solved as in the case of multi-temperature models but with the simultaneous solution of a kinetic equation, as in the case of state-to-state models, but only for the last vibrational level. Then we have presented the self-consistent derivation of a multi-internal temperature model from a vibrational state-tostate model. The objective of the multi-internal temperature model is to describe accurately the vibrational distribution function in using several groups of levels, within which the levels are assumed to follow a Boltzmann distribution at an internal temperature of the group. This multi-internal temperature model has been applied to the simulation of the nonequilibrium coupling between vibration and dissociation behind a shock wave. We have studied the influence of the number of groups on the results and compared, as a reference, with the results obtained with the vibrational stateto-state model. First, using one group, we have shown that assuming a single Boltzmann distribution for all vibrational levels leads to an overestimation of dissociation and consequently to underestimation of temperatures compared with the reference vibrational model. We have shown that the use of a two-internal temperature model was a good compromise between accuracy and complexity for the studied test-case. A thorough study of source terms confirmed that the improvement of the prediction using two groups results from a better prediction first of the global source term $\Omega_{V C}$ and $\Omega_{V T}$, and second of the leveldependence of dissociation, which is required for a good prediction of the chemistry-vibration coupling. Finally, a rovibrational collisional coarse-grain model has been developed to reduce a detailed rovibrational mechanism the $\mathrm{N}_{2}(v, J)+\mathrm{N}$ system to study the internal energy excitation and dissociation processes behind a strong shockwave in a nitrogen flow. It is interesting to note that lessons learned from studying the $\mathrm{N}_{2}(v, J)+\mathrm{N}$ system by means of a coarse-grain model should help to derive in the near future, a reduced mechanism for more complex systems as for example, the $\mathrm{N}_{2}(v, J)+\mathrm{N}_{2}\left(v^{\prime}, J^{\prime}\right)$ system studied in [58].

\section{CONFLICT OF INTEREST}

The authors confirm that this article content has no conflict of interest.

\section{ACKNOWLEDGEMENTS}

The research leading to these results has received funding from the European Community's $7^{\text {th }}$ Framework Programme [FP7/2007/2013] under grant agreement no. 242311. Research of T.E.M. and A.M. is sponsored by the European Research Council Starting Grant \#259354. 


\section{REFERENCES}

[1] Armenise I, Capitelli M, Colonna G, Koudriavtsev N, Smetanin V. Nonequilibrium vibrational kinetics during hypersonic flow of a solid body in nitrogen and its influence on the surface heat-flux. Plasma Chem Plasma Process 1995; 15: 501-28.

[2] Armenise I, Capitelli M, Colonna G, Gorse C. Nonequilibrium vibrational kinetics in the boundary layer of re-entering bodies. J Thermophys Heat Transf 1996; 106: 397.

[3] Kewley DJ. Numerical study of anharmonic diatomic relaxation rates in shock waves and nozzles. J Phys B 1975; 8: 2565.

[4] Colonna G, Tuttafesta M, Capitelli M, Giordano D. Non-arrhenius NO formation rate in one-dimensional nozzle airflow. J Thermophys Heat Transf 1999; 13: 372-5.

[5] Colonna G, Esposito F, Capitelli M. The role of state-selected recombination rates in supersonic nozzle expansion. AIAA Paper 2003-3645, 2003.

[6] Munafò A, Panesi M, Jaffe RL, Colonna G, Bourdon A, Magin TE. QCT-based vibrational collisional models applied to nonequilibrium nozzle flows. Eur Phys J D 2012; 66: 188.

[7] Bruno D, Capitelli M, Esposito F, Longo S, Minelli P. Direct simulation of nonequilibrium kinetics under shock conditions in nitrogen. Chem Phys Lett 2002; 360(1-2): 31-7.

[8] Panesi M, Magin T, Bourdon A, Bultel A, Chazot O. Fire II flight experiment by means of a collisional radiative model. $\mathrm{J}$ Thermophys Heat Transf 2009; 23: 236-48.

[9] Panesi M, Magin T, Bourdon A, Bultel A, Chazot O. Electronic excitation of atoms and molecules for the FIRE II flight experiment. J Thermophys Heat Transf 2011; 25: 361.

[10] Magin T, Panesi M, Bourdon A, Jaffe RL, Schwenke DW. Coarsegrain model for internal energy excitation and dissociation of molecular nitrogen. Chem Phys 2012; 398: 90-5.

[11] Bultel A, Annaloro J. Elaboration of collisional-radiative models for flows related to planetary entries into the Earth and Mars atmospheres. Plasma Sources Sci Technol 2013; 22(2): 25008.

[12] Colonna G, Armenise I, Bruno D, Capitelli M. Reduction of stateto-state kinetics to macroscopic models in hypersonic flows. J Thermophys Heat Transf 2006; 20: 477-86.

[13] Park C. A review of reaction rates in high temperature air. AIAA paper 1989-1740.

[14] Park C. Review of chemical-kinetic problems of future NASA mission, I: earth entries. J Thermophys Heat Transf 1993; 7: 38598.

[15] Sharma SP, Huo WM, Park C. Rate parameters for coupled vibration-dissociation in a generalized $\mathrm{SSH}$ approximation. J Thermophys Heat Transf 1992; 6: 9-21.

[16] Park C. Nonequilibrium Hypersonic Aerothermodynamics. New York: John Wiley \& Sons 1989

[17] Knab O, Frühauf HH, Messerschmid EW. Proceeding of the 2nd European Symposium on Aerothermodynamics for Space Vehicles, ESA Publication Div., Noordwijk. The Netherlands, 129, 1995.

[18] Surzhikov S, Sharikov I, Capitelli M, Colonna G. Kinetic models of nonequilibrium radiation of strong air shock waves. AIAA paper 2006-586, 2006.

[19] Surzhikov S, Rouzaud O, Soubrie T, Gorelov V, Kireev A. Prediction of nonequilibrium and equilibrium radiation for re-entry conditions. AIAA paper 2006-1188, 2006.

[20] Park C. Thermochemical relaxation in shock tunnels. J Thermophys Heat Transf 2006; 20: 689-98.

[21] Kustova EV, Nagnibeda EA, Alexandrova TY, Chikhaoui A. On the nonequilibrium kinetics and heat transfer in nozzle flows. Chem Phys 2002; 276(2): 139-54.

[22] Jaffe R, Schwenke D, Chaban G. Theoretical analysis of $\mathrm{N}_{2}$ collisional dissociation and rotation-vibration energy transfer. AIAA Paper 2009-1569, 2009.

[23] Schwenke D. Dissociation cross-sections and rates for nitrogen. RTO-EN-AVT-162, Nonequilibrium Gas Dynamics, from Physical Models to Hypersonic Flights, Rhode-Saint-Genèse, Belgium, 2008.

[24] Gamallo P, Martínez R, Sayós R, Gonzáles M. Quasiclassical dynamics and kinetics of the $\mathrm{N}+\mathrm{NO} \rightarrow \mathrm{N}_{2}+\mathrm{O}, \mathrm{NO}+\mathrm{N}$ atmospheric reactions. J Chem Phys 2010; 132: 144304.

[25] Esposito F, Capitelli M. QCT calculations for the process $\mathrm{N}_{2}(v)+\mathrm{N}$ $\rightarrow \mathrm{N}_{2}\left(v^{\prime}\right)+\mathrm{N}$ in the whole vibrational range. Chem Phys Lett 2006; 418(4-6): 581-5.
[26] Esposito F, Armenise I, Capitta G, Capitelli M. $\mathrm{O}_{2}-\mathrm{O}$ state-to-state vibrational relaxation and dissociation rates based on quasiclassical calculations. Chem Phys 2008; 351(1-3): 91-8.

[27] Esposito F, Armenise I, Capitelli M. $\mathrm{N}_{2}-\mathrm{N}$ state-to-state vibrational relaxation and dissociation rates based on quasiclassical calculations. Chem Phys 2006; 331(1): 1-8.

[28] Kunc JA, Soon WH. Collisional-radiative nonequilibrium in partially ionized atomic nitrogen. Phys Rev A 1989; 40: 5822-43.

[29] Soon WH, Kunc JA. Thermal nonequilibrium in partially ionized oxygen. Phys Rev A 1990; 41: 825-43.

[30] Bourdon A, Vervisch P. Three-body recombination rate of atomic nitrogen in low-pressure plasma flows. Phys Rev E 1996; 54: 188898.

[31] Bourdon A, Téréziak Y, Vervisch P. Ionization and recombination rates of atomic oxygen in high-temperature air plasma flows. Phys Rev E 1996; 57: 4684-92.

[32] Sawada K, Fujimoto T. Temporal relaxation of excited-level population of atoms and ions in a plasma: validity range of the quasi-steady-state solution of coupled rate equations. Phys Rev E 1994; 49: 5565-73.

[33] Bultel A, van Ootegem B, Bourdon A, Vervisch P. Influence of $\mathrm{Ar}_{2}^{+}$in an argon collisional-radiative model. Phys Rev E 2002; 65(4): 46406

[34] Denbigh K. The principles of chemical equilibrium. Cambridge University Press 1981.

[35] Armenise I, Esposito F, Capitelli M. Dissociation-recombination models in hypersonic boundary layer. J Chem Phys 2007; 336(1): 83-90.

[36] Capitelli M, Armenise I, Gorse C. State-to-state approach in the kinetics of air components under re-entry conditions. J Thermophys Heat Transf 1997; 11: 570-8.

[37] Dunn MG, Kang S-W. Theoretical and experimental studies of reentry plasmas. NASA. Technical Report CR-2232, 1973.

[38] Shatalov OP. Recommended data on rate constants of physical and chemical processes in $\mathrm{N}-\mathrm{O}$ atoms systems. Institute of Mechanics, AVOGADRO Center, Moscow State University, Russia 1987.

[39] Park C, Howe JT, Jaffe RL, Candler GV. Review of chemicalkinetic problems of future NASA missions, II. Mars entries. J Thermophys Heat Transf 1994; 8: 9-23.

[40] Losev SA, Makarov VN, Pogosbekyan MY. Model of the physicochemical kinetics behind the front of a very intense shock wave in air. Fluid Dyn 1995; 30(2): 299-309.

[41] Makarov VN. Determination of the mechanism of physicochemical processes in high-temperature air. J Appl Mech Tech Phys 1996; 37: 206.

[42] Krivonosova OE, Losev SA, Nalivaiko VP, Yu MK, Shatalov OP. Recommended data on the rate constants of chemical reactions among molecules consisting of $\mathrm{N}$ and $\mathrm{O}$ atoms. Plasma Chemistry, Energoizdat, Moscow 1987; p. 1.

[43] Kustova EV, Nagnibeda EA. Deviations from the mass action law in nonequilibrium gas flows. AIP Conf Proc 2005; 762: 908.

[44] Colonna G, Pietanza LD, Capitelli M. Recombination-assisted nitrogen dissociation rates under nonequilibrium conditions. J Thermophys Heat Transf 2008; 22: 399-406.

[45] Colonna G, Pietanza LD, Capitelli M. Reduced two-level approach for air kinetics in recombination regime. AIP Conf Proc 2011; 1333: $1365-70$.

[46] Colonna G, Pietanza LD, Capitelli M. Macroscopic kinetic model for air in nozzle flow. AIP Conf Proc 2012; 1501: 1071.

[47] Krivonosova OE, Shatalov OP. A numerical analysis of rate constants for dissociation of diatomic molecules under quasisteady-state conditions of vibrational nonequilibrium. Chem Phys R 2000; 18(9): 1621-30.

[48] Bose D, Candler GV. Thermal rate constants of the $\mathrm{O}_{2}+\mathrm{N} \rightarrow \mathrm{NO}+\mathrm{O}$ reaction based on the ${ }^{2} A^{\prime}$ and ${ }^{4} A^{\prime \prime}$ potential energy surfaces. J Chem Phys 1997; 107(16): 6136-45.

[49] Bose D, Candler GV. Thermal rate constants of the $\mathrm{N}_{2}+\mathrm{O} \rightarrow \mathrm{NO}+\mathrm{N}$ reaction using ab initio ${ }^{3} A^{\prime \prime}$ and ${ }^{3} A^{\prime}$ potential energy surfaces. J Chem Phys 1996; 104(8): 2825-33.

[50] Colonna G, Tuttafesta M, Capitelli M, Giordano D. Influence of $\mathrm{O}_{2}(v)+\mathrm{N} \rightleftharpoons \mathrm{NO}+\mathrm{O}$ on $\mathrm{NO}$ formation rate in one-dimensional air nozzle flow. J Thermophys Heat Transf 2000; 14(3): 455-6.

[51] Guy A, Bourdon A, Perrin MY. Derivation of a consistent multiinternal-temperature model for vibrational energy excitation and 
dissociation of molecular nitrogen in hypersonic flows. AIAA paper 2013-0194, 2013.

[52] Guy A, Bourdon A, Perrin MY. Consistent multi-internaltemperatures models for nonequilibrium nozzle flows. Chem Phys 2013; 420: 15-24

[53] Le Roy RJ, Huang Y, Jary C. An accurate analytic potential function for ground state $\mathrm{N}_{2}$ from a direct potential fit analysis of spectroscopic data. J Chem Phys 2006; 125: 164310.

[54] Adamovich VI, Macheret SO, Rich WJ, Treanor CE. Vibrational relaxation and dissociation behind shock waves. 1: Kinetic rates model. AIAA J 1995; 33(6): 1064-9.
[55] Adamovich VI, Macheret SO, Rich WJ, Treanor CE. Vibrational relaxation and dissociation behind shock waves. 2: Master equation modeling. AIAA J 1995; 33(6): 1070-5.

[56] Panesi M, Jaffe RL, Schwenke DW, Magin TE. Rovibrational internal energy transfer and dissociation of $\mathrm{N}_{2}\left({ }^{1} \Sigma_{g}^{+}\right)-\mathrm{N}\left({ }^{4} S_{u}\right)$ system in hypersonic flows. J Chem Phys 2013; 138: 044312.

[57] Annaloro J, Morel V, Bultel A, Omaly P. Global rate coefficients for ionization and recombination of carbon, nitrogen, oxygen, and argon. Phys Plasmas 2012; 19: 73515.

[58] Jaffe R, Schwenke D, Chaban G. Vibration-rotation excitation and dissociation in $\mathrm{N}_{2}-\mathrm{N}_{2}$ collisions from accurate theoretical calculations. AIAA Paper 2010-4517, 2010.

(C) Bourdon et al.; Licensee Bentham Open.

This is an open access article licensed under the terms of the Creative Commons Attribution Non-Commercial License (http://creativecommons.org/licenses/by-nc/3.0/) which permits unrestricted, non-commercial use, distribution and reproduction in any medium, provided the work is properly cited. 